\title{
Dynamic heterogeneities in the out-of-equilibrium dynamics of simple spherical spin models
}

\author{
Gregor Diezemann \\ Institut für physikalische Chemie, Universität Mainz, Welderweg 11, 55099 Mainz, FRG \\ revised version
}

\begin{abstract}
The response of spherical two-spin interaction models, the spherical ferromagnet (s-FM) and the spherical Sherrington-Kirkpatrick (s-SK) model, is calculated for the protocol of the so-called nonresonant hole burning experiment (NHB) for temperatures below the respective critical temperatures. It is shown that it is possible to select dynamic features in the out-ofequilibrium dynamics of both models, one of the hallmarks of dynamic heterogeneities. The behavior of the s-SK model and the s-FM model in three dimensions is very similar, showing dynamic heterogeneities in the long time behavior, i.e. in the aging regime. The appearence of dynamic heterogeneities in the s-SK model explicitly demonstrates that these are not necessarily related to spatial heterogeneities. For the s-FM it is shown that the nature of the dynamic heterogeneities changes as a function of dimensionality. With incresing dimension the frequency selectivity of the NHB diminishes and the dynamics in the meanfield limit of the s-FM model becomes homogeneous.
\end{abstract}

PACS Numbers: 64.70 Pf,05.40.+j,61.20.Lc

\section{Introduction}

Non-exponential relaxation behavior is found to be rather common when dealing with disordered materials like glasses, spin-glasses, disordered crystals or proteins [1]. In the last decade particular attention has been payed to the question to which extent the relaxation is to be viewed as dynamic heterogeneous [2]. Different experimental techniques have been invented in order to investigate the detailed nature of the relaxation particularly of amorphous systems [3, 4, 5, 6]. These techniques allow to specifically select a slow sub-ensemble and afterwards monitor its relaxation. Various applications of these techniques have shown that the primary response in amorphous polymers and supercooled liquids is to be viewed as heterogeneous in the sense that it is possible to select slow sub-ensembles relaxing at smaller rates than the average. Throughout this paper I use the definition given in ref. [7] 
according to which a system will be called dynamic heterogeneous if it is possible to select dynamically distinguishable (slow and/or fast) contributions to a relaxation.

In addition it has been found that after a certain re-equilibration time the relaxation properties of the selected sub-ensemble return to those of the bulk [8, 9]. Therefore, these experiments indicate that the response can be described as a superposition of exponentially decaying entities with different relaxation rates. The various relaxation rates, however, are not static quantities but apparently fluctuate in time. Different interpretations have been provided for this behavior [10, 11. The NMR-techniques [8, 9] have the advantage of a simple interpretation in terms of equilibrium 4-time correlation functions but are restricted to a rather narrow temperature regime and to certain materials. The optical deep bleach technique 4. has the advantage that it can be applied in a wider temperature range with the shortcoming that up to now it has not been interpreted in terms of equilibrium correlation functions. At this point it is important to note that these experimental methods monitor molecular reorientations and therefore are not able to address the question of spatial aspects of the dynamic heterogeneities. The only exception is provided by a new variant of the 4d-NMR technique 12, which allows to extract a length scale via monitoring spin-diffusion. Originally, this technique has been applied to a polymeric liquid and later on the length scale of the dynamic heterogeneities has been extracted for low-molecular glass-forming systems [13, 14]. The corresponding length scales have been found to be on the order of $1 \cdots 4 \mathrm{~nm}$.

Another experimental technique allowing to monitor dynamic heterogeneities is provided by the nonresonant spectral hole burning (NHB) experiment 15. This method is based on a pump-wait-probe field sequence with a large pump field amplitude beyond the linear response regime. Though originally applied to supercooled liquids[16], NHB in the meantime has been used to investigate the relaxation of several materials, including disordered crystals (relaxor ferroelectrics) [17, ion-conducting glasses 18, and spin glasses 19. Also the application of NHB to a solvable glass-model has been presented [20]. The interpretation of the obtained results has mainly been guided by the fact that via the application of a large amplitude ac field of frequency $\Omega$ the sample absorbs energy of an amount proportional to the imaginary part of the susceptibility evaluated at the pump frequency $\Omega$ 21. In case of a homogeneously broadened response one does not expect that it is possible to modify the response in a frequency selective way. By contrast such a goal could be achieved if the response is given by a superposition of differently fast relaxing entities (heterogeneous scenario). This is because in this case energy absorbtion should be largest for those sub-ensembles with a relaxation time on the scale of the inverse pump frequency. This intuitive picture is confirmed in the framework of a response-theory for NHB for the particular case of stochastic dipole reorientations, which I have developed recently [22].

In the quoted experiments it always has been found that a frequency selective modification of the response indeed is possible. Regarding the re-equilibration of this modification, however, the results differ not only with respect to the frequency dependence but also regarding the time scale of the recovery. For the latter a time scale longer than the inverse burn frequency has been observed in the case of the relaxor ferroelectrics 17. 
As long as one is concerned with supercooled liquids, one can safely consider the system to be in (metastable) equilibrium prior to the NHB field sequence. This, however, is not necessarily true for the relaxor materials or the spin glasses. In particular, it was argued in ref. 23] that the results obtained for the solvable p-spin-glass model 20] are mainly to be interpreted in terms of out-of-equilibrium effects. In equilibrium, the $p=3$-model studied shows an exponential relaxation at long times. Therefore, according to what was said above one does not expect to be able to select a sub-ensemble in a frequency dependent way in the equilibrated version of the model. It has to be mentioned here that dynamic heterogeneities as monitored by NHB have also been observed in Monte Carlo simulations on an equilibrated Sherrington-Kirkpatrick mean-field spin-glass model 24]. These calculations explicitly demonstrate that from the observation of dynamic heterogeneities one cannot conclude on the existence of spatial heterogeneities. Also, this finding appears to be independent of whether the system was in thermal equilibrium before the application of the pump-field.

In this paper I consider the application of the NHB field sequence to the spherical Sherrington-Kirkpatrick (s-SK) model, i.e. the p-spin model with $p=2$ and the spherical ferromagnet (s-FM) in arbitrary dimension. The Langevin dynamics for these models has been solved analytically 25]. Additionally, the s-FM model is equivalent to the $\mathrm{O}(\mathrm{N})$-model in the limit of large $\mathrm{N}$ and therefore is a typical model for domain coarsening processes [26].

I will solely consider a thermal history protocol in which the system is quenched to a temperature below the critical temperature $T_{c}$ from infinite temperature prior to the experiment. The behavior at and above $T_{c}$ will be investigated in a forthcoming publication. Therefore, all effects observed are intimately related to the out-of-equilibrium dynamics of the model. This is because the system never reaches equilibrium in this temperature regime. The outline of the paper is as follows. In the next Section I will briefly recall the dynamic features of the models and calculate the response to the NHB field sequence in second order regarding the pump-field amplitude and linearly in the small step-field. In Section III the results of the calculations are presented and discussed. The paper closes with some conclusions in Section IV.

\section{NHB in spherical two-spin interaction models}

The spherical models under consideration are defined by the Hamiltonian

$$
\mathbf{H}=-\frac{1}{2} \sum_{i \neq k} J_{i k} s_{i} s_{k}-\sum_{i} h_{i} s_{i}
$$

where in case of the s-SK model the $J_{i k}$ are chosen at random from a Gaussian probability distribution with zero mean and variance $\sigma=1 / N$ and are restricted to a ferromagnetic coupling $J$ (to be set to unity in the following) for the s-FM model on a simple hypercubic lattice in d dimensions. In addition the spin-variables are subject to the spherical constraint 
$\sum_{i} s_{i}^{2}=N$. The Langevin equations governing the dynamics of the model read as

$$
\dot{s}_{i}(t)=\sum_{k} J_{i k} s_{k}(t)+h_{i}-z(t) s_{i}(t)+\xi_{i}(t)
$$

where $z(t)$ is the Lagrange multiplier enforcing the spherical constraint and $\xi_{i}(t)$ is a $\delta$ correlated Gaussian white noise. These equations have been solved analytically by Cugliandolo and Dean (CD) 25] in two papers. The dynamical properties of the s-FM model are discussed in ref.[27]. Further information regarding the correspondence between the two models can be found in ref. 28. Here, I briefly summarize the results relevant in the present context.

Of particular importance are the violations of the fluctuation-dissipation theorem (FDT), which relates the response function to the time-derivative of the two-time correlation function,

$$
R(\tau)=-\frac{1}{T} \frac{d C(\tau)}{d \tau}
$$

In particular, it has proven extremely useful in out-of -equilibrium situations to define the so-called fluctuation-dissipation ratio $X\left(t, t_{w}\right)$ via[29],

$$
R\left(t, t_{w}\right)=\frac{X\left(t, t_{w}\right)}{T} \frac{\partial C\left(t, t_{w}\right)}{\partial t_{w}}
$$

the limiting long-time behavior, $X_{\infty}$, of which is known to vanish for domain coarsening models [30. Here, $t_{w}$ denotes the time that has elapsed after a quench to the working temperature prior to the measurement. Also for the models considered in the present paper one has $X_{\infty}=0$ [27]. Thus, concerning this measure of typical distances from equilibrium, the domain coarsening models in finite dimension do not show any differences to the mean-field s-SK model.

The response of the system, $R_{h}\left(t, t^{\prime}\right)=\sum_{i}^{N}\left\langle s_{i}(t) \xi_{i}\left(t^{\prime}\right)\right\rangle /(2 N T)$, in the presesence of a field $h(t)$ can be obtained in the same way as calculated by CD for the zero field case. As shown by Berthier et al. 31] this yields:

$$
R_{h}\left(t, t^{\prime}\right)=\theta\left(t-t^{\prime}\right) \frac{W_{h}\left(t^{\prime}\right)}{W_{h}(t)} g\left(\frac{t-t^{\prime}}{2}\right)
$$

where the function $g(t)$ is defined by

$$
\begin{aligned}
& g(t)=\left[\exp (-4 t) I_{0}(4 t)\right]^{d} \quad \mathrm{~s}-\mathrm{FM} \\
& g(t)=\exp (-4 t) \frac{I_{1}(4 t)}{2 t} \quad \mathrm{~s}-\mathrm{SK}
\end{aligned}
$$

with $I_{n}(x)$ denoting the generalized Bessel function and $W_{h}(t)$ is the solution of

$$
\begin{aligned}
W_{h}(t)^{2}= & g(t)+2 T \int_{0}^{t} d \tau W_{h}(\tau)^{2} g(t-\tau) \\
& +\int_{0}^{t} d t_{1} \int_{0}^{t} d t_{2} h\left(t_{1}\right) h\left(t_{2}\right) W_{h}\left(t_{1}\right) W_{h}\left(t_{2}\right) g\left(t-\frac{t_{1}+t_{2}}{2}\right)
\end{aligned}
$$

which has its origin in the normalization of the equal-time correlation, $C(t, t)=1$, i.e. the spherical constraint. 


\section{Zero field response}

Before I turn to the calculation of the response following the NHB pulse sequence, it is appropriate to summarize the known results for the reponse and the correlation in zero field, for a more detailed discussion see refs. [25, 27, 28]. In zero field, eq.(77) simplifies to the following Volterra equation:

$$
W(t)^{2}=g(t)+2 T \int_{0}^{t} d \tau W(\tau)^{2} g(t-\tau)
$$

For the s-SK model, this equation has been solved by CD for random initial conditions (i.e. a quench from $T=\infty$ at $t=0$ ). No simple analytical solution exists for the s-FM model. However, for $t \gg 1$ it can be shown[25, 27, 28, that for $T<T_{c}$

$$
W(t)^{2}=\frac{1}{\left(1-T / T_{c}\right)^{2}} g_{a s}(t)
$$

Here, the asymptotic behavior of the $g(t)$ are given by

$$
\begin{array}{ll}
g_{a s}(t)=(8 \pi t)^{-d / 2} & \text { s-FM } \\
g_{a s}(t)=(32 \pi)^{-1 / 2} t^{-3 / 2} & \text { s-SK }
\end{array}
$$

The critical temperatures are given by $T_{c}=1$ for the s-SK model and $T_{c}$ depends on the spatial dimension in case of the s-FM model, with $T_{c}(d=3) \simeq 3.9568[32$. For the other dimensions used in the present paper I find $T_{c}(d=5) \simeq 8.6482, T_{c}(d=7) \simeq 12.7982$ and $T_{c}(d=9) \simeq 16.8579$.

If $t_{w}$ denotes the time elapsed after a quench from $T=\infty$ to $T<T_{c}$, the following behavior is found for $R\left(t+t_{w}, t_{w}\right)$ from eqns.(5910) in the limit of long $t_{w}$ :

$$
\begin{gathered}
R\left(\tau+t_{w}, t_{w}\right)=\lambda^{-\epsilon / 4} g(\tau / 2) \quad \text { with } \quad \lambda=\frac{t_{w}}{t_{w}+\tau} \quad ; \quad t_{w} \gg 1 \\
\epsilon=d \quad(\mathrm{~s}-\mathrm{FM}) \quad ; \quad \epsilon=3 \quad(\mathrm{~s}-\mathrm{SK})
\end{gathered}
$$

From this expression it is evident already that the dynamic properties of the s-SK model are very similar to those of the s-FM in $d=3$. Therefore, the following expressions are given for the s-FM model. The only differences between the s-FM model in $d=3$ and the s-SK model stem from the different prefactors in eq.(10). In particular, it is evident from eq.(11) that the temperature is an irrelevant variable in the whole low-temperature phase.

Two time-sectors are to be distinguished:

- For short times $\tau$ such that $\tau \ll t_{w}$ - the so-called stationary regime - one has $\lambda \simeq 1$ and accordingly:

$$
R\left(\tau+t_{w}, t_{w}\right)=R(\tau)=g(\tau / 2) \quad \tau \ll t_{w}
$$

In particular, in this stationary regime the FDT, eq.(3), holds. In the long time limit, $1 \ll \tau$, eq.(12) shows that $R(\tau)$ decays according to $R(\tau) \sim \tau^{-d / 2}$. 
- In the so-called aging regime $1 \ll \tau \sim t_{w}$, one finds from eq.(11):

$$
R\left(\tau+t_{w}, t_{w}\right)=\lambda^{-d / 4}(4 \pi \tau)^{-d / 2} \quad 1 \ll \tau \sim t_{w}
$$

In this regime the FDT is strongly violated. If in addition $\tau \gg t_{w}$, the response behaves as $R\left(\tau, t_{w}\right) \sim \tau^{-d / 4}$.

The overall behavior is shown for various values of the waiting time in Fig.1. From this plot it is seen that for all dimensions shown, $d=3,5,7$, there is a crossover from the $\tau^{-d / 2}$ to the $\tau^{-d / 4}$ behavior. Also the explicit dependence on the waiting time in the aging regime is evident. It should be pointed out that the crossover from the stationary regime to the aging (domain growth) regime takes place around $\tau \sim t_{w}$ independent of spatial dimension. Remember, that only in the stationary regime the FDT holds. In particular, the behavior of the response (and also the two-time correlation function) does not change qualitatively around $d=4$, above which the model behaves mean-field like concerning the statics and the exponents. (The same holds for the dynamic fluctuations, as will be shown elsewhere.)

The above discussion shows that the relaxation is extremely non-exponential. Therefore, the question as to what extent the response can be viewed as dynamic heterogeneous naturally arises.

\section{Nonresonant hole burning}

In the following, the response will be calculated for the NHB-field-sequence, cf. Fig.2. At $t=0$ the system is quenched from $T=\infty$ to the working temperature $T$. One (or more) cycles of the pump-field $h_{p}(t)=h_{p} \sin \left[\Omega\left(t-t_{q}\right)\right]$ are applied after a time $t_{q}$ has elapsed. Following a waiting time $t_{w}$ the response $R^{*}\left(\hat{t}+\tau, \hat{t}+\tau^{\prime}\right)$ is measured, where I defined

$$
\hat{t}=t_{q}+t_{p}+t_{w} \quad \text { and } \quad t_{p}=2 N \pi / \Omega
$$

for brevity. Here, $N$ denotes the number of cycles of the sinusoidal pump-field. In the following calculations of $R^{*}$ the $W_{h}(t)$ are needed in second order with respect to the pump-field amplitude $h_{p}$. From eq.(17) it is evident that a perturbation expansion follows from

$$
W_{h_{p}}(t)^{2}=W(t)^{2}+h_{p}^{2} \Delta(t)^{2}+\mathcal{O}\left(h_{p}^{4}\right)
$$

Inserting this expression into eq.(17) yields eq.(8) for the zero'th order term and, assuming a time-dependent field of the form $h(t)=h_{p} \sin \left[\Omega\left(t-t_{q}\right)\right] \theta\left(t-t_{q}\right)$ according to Fig.2:

$$
\begin{aligned}
& \Delta\left(t_{q}+\tau\right)^{2}=2 T \int_{0}^{\tau} d s \Delta\left(t_{q}+s\right)^{2} g(\tau-s)+\Delta_{0}\left(t_{q}+\tau\right) \\
& \Delta_{0}\left(t_{q}+\tau\right)=\int_{0}^{t_{m}} d t_{1} \int_{0}^{t_{m}} d t_{2} \sin \left(\Omega t_{1}\right) \sin \left(\Omega t_{2}\right) W\left(t_{q}+t_{1}\right) W\left(t_{q}+t_{2}\right) g\left(\tau-\frac{t_{1}+t_{2}}{2}\right)
\end{aligned}
$$


where $t_{m}=\operatorname{Min}\left(\tau, t_{p}\right)$. Using eq.(14) the response function in $\mathcal{O}\left(h_{p}^{2}\right)$ then is found to be given by $\left(\tau>\tau^{\prime}\right)$ :

$$
\begin{aligned}
& R^{*}\left(\hat{t}+\tau, \hat{t}+\tau^{\prime}\right)=R\left(\hat{t}+\tau, \hat{t}+\tau^{\prime}\right)+\Delta R\left(\hat{t}+\tau, \hat{t}+\tau^{\prime}\right) \\
& R\left(\hat{t}+\tau, \hat{t}+\tau^{\prime}\right)=\frac{W\left(\hat{t}+\tau^{\prime}\right)}{W(\hat{t}+\tau)} g\left(\frac{\tau-\tau^{\prime}}{2}\right) \\
& \Delta R\left(\hat{t}+\tau, \hat{t}+\tau^{\prime}\right)=-\frac{h_{p}^{2}}{2}\left[\frac{\Delta(\hat{t}+\tau)^{2}}{W(\hat{t}+\tau)^{2}}-\frac{\Delta\left(\hat{t}+\tau^{\prime}\right)^{2}}{W\left(\hat{t}+\tau^{\prime}\right)^{2}}\right] R\left(\hat{t}+\tau, \hat{t}+\tau^{\prime}\right)
\end{aligned}
$$

In the NHB-protocol of Fig.2, however, the response to a small step field is recorded, i.e. the integrated response (the thermoremanent magnetization):

$$
\chi^{*}(\hat{t}, \tau)=\chi(\hat{t}, \tau)+\Delta \chi(\hat{t}, \tau)=\int_{0}^{\tau} d s R^{*}(\hat{t}+\tau, \hat{t}+s)
$$

according to eq.(16) with the zero-field integrated response $\chi(\hat{t}, \tau)=\int_{0}^{\tau} d s R(\hat{t}+\tau, \hat{t}+s)$. Eqns.(15) …(17) allow the calculation of the results of a NHB experiment at any desired temperature.

\section{Results and discussion}

Many of the general features of the modification $\Delta \chi(\hat{t}, \tau)$ can already be seen for $T=0$, which is the simplest case. Afterwards finite temperatures will be discussed as well as the dependence of the observed features on spatial dimension. Finally, a direct comparison between the three-dimensional s-FM model and the s-SK model will by carried out.

Throughout the remainder of the paper the dependence on $\hat{t}$ will be skipped whenever no confusion can occur, i.e. the shorthand notation $\chi(\tau) \equiv \chi(\hat{t}, \tau)$ and $\Delta \chi(\tau) \equiv \Delta \chi(\hat{t}, \tau)$ will be used. Times and frequencies will be given in dimensionless units.

\section{A. Spherical ferromagnet}

In this subsection the details of the results for calculations of the response following the NHB field sequence are discussed. The discussion is kept general with regard to spatial dimension but the actual calculations are carried out for $d=3$, cf. Figs.3 $\cdots 6$. The dependence of the results on spatial dimension will be presented in the next subsection.

$\mathbf{T}=\mathbf{0}:$

For $T=0$, eq.(15) can be solved trivially and the modified response is easily calculated. From eq.(8) one explicitly has

$$
W(t)^{2}=g(t) \quad \text { and } \quad \Delta(t)^{2}=\Delta_{0}(t) \quad ; \quad T=0
$$

The corresponding expressions for $\chi(\tau)$ and $\Delta \chi(\tau)$ are easily obtained from eqns. (16 17). 
From the discussion of the zero field response in the last section it is evident that the time $t_{q}$ elapsed after the quench and before application of the pump-field is a very important parameter. For small $t_{q}$ some transient features are expected due to the interplay of the approach of the aging-regime and the additional non-equilibrium features induced by the application of the pump-field. Of course, the cross-over to a $\Omega$ independent behavior will depend crucially on the pump-frequency $\Omega$ as this determines the time $t_{p}$ of the imposed non-equilibrium situation. This is demonstrated in Fig.3a, where $\Delta \chi(\tau)$ is plotted for $d=3, T=0, t_{w}=0, \Omega=0.1,10$ and several values of $t_{q}$. From this figure two features are evident immediately. First of all it is seen that $\Delta \chi(\tau)$ is non-zero only in a limited time interval and the time of the maximum modification depends on the burn frequency $\Omega$, thus demonstrating dynamic heterogeneous behavior. Additionally, the curves for $t_{q}=0$ differ from the others in that they change from positive to negative values in a limited time range. This transient behavior also depends on $\Omega$. However, it is always possible to choose $t_{q}$ in a way that the mentioned interplay between the two sources of transient features can be neglected. Thus, it is interesting to consider the asymptotic regime determined by

$$
t_{q} \gg 1 \text { where } t_{q}=t_{q}(\Omega)
$$

The detailed dependence of $t_{q}$ on $\Omega$ has to be found empirically in the sense that no transient effects should show up in the modified response for a given pump frequency $\Omega$. In order to further demonstrate the relative independence of the results from the chosen value of $t_{q}$, in Fig.3b I have plotted the value of the maximum modification, $\Delta \chi_{\max }=\Delta \chi\left(\tau_{\max }\right)$ vs. $t_{q}$ for $\Omega=0.01,0.1,10$ in a scaled way. This plot demonstrates the features already mentioned above. For small $t_{q}$ there is some time interval in which $\Delta \chi(\tau)$ is negative and therefore the maximum is reduced. The small hump around $t_{q} \sim 2 \pi / \Omega$ in the curves is roughly located at those values of $t_{q}$ where also the negative part vanishes. Finally for long $t_{q}$ a plateau is observed and the results are independent of $t_{q}$. Obviously, the minimum value of $t_{q}$ satisfying the constraint that $\Delta \chi(\tau)$ is independent of $t_{q}$ depends on $\Omega$. In the whole range of $\Omega$ considered in the present paper it turned out that a value of $t_{q}=10^{6}$ is sufficient. Therefore all further calculations are performed for $t_{q}=10^{6}$ unless stated otherwise. (I have checked via explicit calculations that $\chi\left(t_{q}+t_{p}, \tau\right)-\chi\left(t_{q}, \tau\right) \simeq 0$ independent of $\Omega$. Otherwise, the interpretation of the $\Omega$-dependence of $\Delta \chi(\hat{t}, \tau)$ in terms of dynamic heterogeneities would be meaningless.) From eqns.(10) and (15) it is seen that in this regime one has for $\Delta_{0}\left(t_{q}+\tau\right)$ for arbitrary dimension $\left(t_{m}=\operatorname{Min}\left(\tau, t_{p}\right)\right)$ :

$$
\Delta_{0}\left(t_{q}+\tau\right)=(8 \pi)^{-d / 2} \int_{0}^{t_{m}} d t_{1} \int_{0}^{t_{m}} d t_{2} \sin \left(\Omega t_{1}\right) \sin \left(\Omega t_{2}\right) \frac{g\left(\tau-\frac{t_{1}+t_{2}}{2}\right)}{\left[\left(t_{q}+t_{1}\right)\left(t_{q}+t_{2}\right)\right]^{d / 4}} \quad t_{q} \gg 1
$$

This expression along with eq.(16) for the modification of the response also explains the observed relatively weak dependence of $\Delta \chi(\tau)$ on $t_{q}$. In lowest order $\Delta_{0}\left(\hat{t}+\tau^{\prime}\right)$ behaves as $t_{q}^{-d / 2}$ and according to eq.(9) the same holds for $W\left(\hat{t}+\tau^{\prime}\right)$. Therefore, $R$ and $\Delta R$ are independent of $t_{q}$ in this order and the same holds for $\Delta \chi$. An explicit $t_{q}$-dependence enters only in higher order. 
For $T=0$, the above expression is only needed for $t_{m}=t_{p}=2 N \pi / \Omega$, where $N$ denotes the number of cycles of the sinusoidal field. The reason for the fact that smaller values of $t_{m}$ are irrelevant in this case is that according to eq.(18) $\Delta(t)^{2}=\Delta_{0}(t)$ and that the response is measured only after the pump-field is switched off. Therefore, the question as to which extent the results depend on the number of cycles naturally occurs. Fig.3c shows $\Delta \chi(\tau)$ as a function of $\tau$ for $N=1,5,10$ for $\Omega=0.1$. The curves for $N=5$ and $N=10$ are hardly distinguishable in this plot. The saturation of the maximum amplitude is demonstrated in Fig.3d, where $\Delta \chi_{\max }$ is plotted vs. $N$ for $\Omega=0.1$ and $10\left(t_{q}=10^{6}\right)$. For $N$ values larger than roughly $6, \Delta \chi_{\max }$ become $N$-independent. In all of these calculations one has $t_{q} \gg t_{p}$, whereas the opposite limit is met at small $t_{q}$ as discussed above, although for $N=1$. This demonstrates that the field sequence of NHB is unable to drive the system much further away from equilibrium than it already is due to the quench at $t=0$.

After this consideration of the influence of the parameters $t_{q}$ and $N$ now the more important issue of the $\Omega$-dependence of $\Delta \chi(\tau)$ will be discussed. Before presenting the results of model calculations it is instructive to investigate analytically the limiting behavior of $\Delta_{0}\left(t_{q}+\tau\right)$ according to eq.(19) which in turn determines the behavior of $\Delta \chi(\tau)$. One finds that $\Delta_{0}$ vanishes in the limits of large and small $\Omega$ according to

$$
\begin{aligned}
& \Omega \rightarrow 0: \quad \Delta_{0}(\hat{t}+\tau) \sim \Omega^{d / 2-2} \quad\left(\Omega t_{q} \gg 1\right) \\
& \Delta_{0}(\hat{t}+\tau) \sim \Omega^{d-2} \quad\left(\Omega t_{q} \ll 1\right) \\
& \Omega \rightarrow \infty: \quad \Delta_{0}(\hat{t}+\tau) \sim \Omega^{-4} \quad \forall \mathrm{d}
\end{aligned}
$$

demonstrating that the modification induced by the NHB pulse sequence in principle depends on $\Omega$. The universal $\Omega^{-4}$-dependence for large $\Omega$ can easily be understood by slightly rewriting eq.(15) in the form $\left(M \equiv \Omega t_{m}\right)$

$$
\Delta_{0}\left(t_{q}+\tau\right)=\Omega^{-2} \int_{0}^{M} d t_{1} \int_{0}^{M} d t_{2} \sin t_{1} \sin t_{2} W\left(t_{q}+\frac{t_{1}}{\Omega}\right) W\left(t_{q}+\frac{t_{2}}{\Omega}\right) g\left(\tau-\frac{t_{1}+t_{2}}{2 \Omega}\right)
$$

The behavior for large $\Omega$ is obtained via second-order expansions of $W\left(t_{q}+\Omega^{-1} x\right)$ and of $g\left(\tau-\Omega^{-1} x\right)$. In a simple calculation one then finds the quoted $\Omega^{-4}$ behavior. Therefore, this represents a universal result which is valid for any model obeying eq. (15). As the $\Omega$ dependence of $\Delta_{0}\left(t_{q}+t\right)$ uniquely determines the one of $\Delta\left(t_{q}+t\right)^{2}$, this result additionally holds for all temperatures, including the disordered paramagnetic phase.

In Fig. 4 a the dependence of $\Delta \chi(\tau)$ on $\Omega$ is demonstrated in detail for $d=3$. It is clearly seen that $\Delta \chi(\tau)$ shows a very pronounced $\Omega$-dependence for $\Omega<0.1$ which, however, diminishes with increasing $\Omega$. Additionally, it is evident that the spectral modifications become 'broader', i.e. are non-vanishing in a larger time interval, as $\Omega$ decreases.

In Fig.4b I have plotted the time of the maximum modification, $\tau_{\max }$ vs. $\Omega$. From this plot one can see that $\tau_{\max }$ varies as $\Omega^{-1}$ for small $\Omega$. This is the behavior typical for an extremely broad distribution of relaxation times. Additionally, $\tau_{\max }$ becomes independent of $\Omega$ for $\Omega \sim 10$. Thus, for the s-FM model in $d=3$, the response is dynamic homogeneous in the short time regime, whereas it is dynamic heterogeneous for long times. Therefore, 
the aging dynamics in this model is dynamic heterogeneous. Also, it is seen from Fig.4b that the behavior is independent of the time $t_{q}$ elapsed after the quench.

Another important question regards the time scale of the 'recovery' of the modification, i.e. the waiting time dependence. In ref. 22] I have found that in a model of reorientational dynamics there is no extra time scale for the recovery. However, in the experiments on the relaxor materials a very long recovery time scale has been found[17]. In order to investigate this question, in Fig.5a I have plotted the maximum modification (normalized to the value at $\left.t_{w}=0\right), \Delta \chi\left(\tau_{\max }\right)_{n o r m}$. vs. scaled waiting time $\Omega t_{w}$ for a variety of burn frequencies $\Omega$. It is evident that the life-time strongly depends on $\Omega$ for small $\Omega$ and that this dependence diminishes for larger $\Omega$. It has to be mentioned at this point that the form of the modifications hardly change for different waiting times. In order to have a simple measure for the recovery times, I have fitted the curves of Fig.5a to a Kohlrausch function, $\exp \left\{-\left(t_{w} / \tau_{K}\right)^{\beta_{K}}\right\}$, and plotted the resulting time scales $\tau_{K}$ vs. $\Omega$ in Fig.5b. I included the time of the maximum modification for $t_{w}=0$ (cf. Fig.4b) in this plot (dot-dashed line). As the behavior of these two time scales is almost identical to within a factor of two, the conclusion to be drawn from these calculations is that there does not exist an extra time scale for the recovery in the s-FM model. This appears plausible on intuitive grounds because the only time scale set by the pump is roughly $1 /(2 \pi \Omega)$ in the domain growth regime and just unity $(1 /(2 \pi))$ in the short-time regime. This is qualitatively different from a complex domain-structured system like the relaxor materials, where pinning effects play a dominant role.

\section{$\mathbf{T}>0$ :}

So far, I have considered $T=0$ solely. As already mentioned in the last section, the response in the ferromagnetic phase is independent of temperature. For the NHB field sequence, however, eqns.(15) and (16) show that $\Delta R$ and correspondingly also $\Delta \chi(\tau)$ do depend on temperature. The physical reason for this dependence is quite clear. Though the thermal noise is irrelevant for the linear response, this changes under NHB conditions due to the aligning effect of the pump-field. Here, thermal fluctuations tend to counterbalance the induced alignment.

In order to investigate the importance of this effect, eq.(15) is solved numerically for finite temperatures for $t_{q} \gg 1$, i.e. using eq.(9) in the expression for $\Delta_{0}(t)$. (To ensure that the physical solution of the equation is met, I first performed calculations for very small $\mathrm{T}$, in which case eq.(15) can be solved in terms of an expansion in $T / T_{c}$.) The results of such temperature dependent calculations are shown in Fig.6a, where $\Delta \chi(\tau)$ is plotted for various $\Omega$ and for temperatures up to $0.5 T_{c}$ using $t_{q}=10^{6}$ and $t_{w}=0$. Two features are evident by inspection of that plot. First, the position of the maximum modification hardly changes as a function of temperature. It should be mentioned that also the shape of the $\Delta \chi(\tau)$ does not change as a function of temperature. This means that no effect of 'motional narrowing' is observable.

The most prominent feature is the increasing intensity of $\Delta \chi(\tau)$. In order to demon- 
strate this behavior in more detail, the intensity at the time of the maximum modification, $\Delta \chi\left(\tau_{\max }\right)$, is shown as a function of reduced temperature $1-T / T_{c}$ in Fig.6b for $\Omega=0.01,100$. This plot demonstrates a scaling behavior of these quantities. The exponents, however, are different for various frequencies and do not seem to have an obvious explanation. It would be interesting to further analyze the $\Omega$-dependence of $\Delta \chi\left(\tau_{\max }\right)$, which, however, is beyond the scope of the present study.

The conclusion to be drawn from these calculations is that the effect of temperature is seen mainly in the amplitude of the modification, but narrowing effects are absent. This means that the nature of the dynamic heterogeneities are not affected by temperatureeffects. Therefore, in the following, I will concentrate on $T=0$ for simplicity.

\section{B. Spherical-ferromagnet: NHB for varying dimension}

In contrast to the s-SK model the s-FM model offers the opportunity to study the behavior of the observed dynamic heterogeneities as a function of spatial dimension. As already mentioned in Sect.II the fluctuation-dissipation ratio $X_{\infty}$ vanishes for the s-FM model independent of spatial dimension. Additionally, it is known 27] that the usual static critical exponents take on their mean-field values for $d>4$ while some dynamical exponents depend on dimension for arbitrary $d$. The only hint for a change in the dynamic properties of coarsening models for $d>4$ stems from the large N-model, where it has been shown that the aging contribution to the integrated response changes qualitatively for $d>4[33$. The two-time quantities in the spherical models considered here, however, do not show any signature of a change in behavior around $d=4$. Therefore, in this subsection, the dependence of the behavior of $\Delta \chi(\tau)$ and thus of the dynamic heterogeneities on spatial dimension will be investigated for $T=0$.

Fig.7a shows a plot of $\Delta \chi(\tau)$ versus $\tau$ for $d=5$ (upper panel) and $d=7$ (lower panel), which is to be compared to Fig.4a. (I do not consider even spatial dimensions like $d=6$, because the calculations are much more involved for technical reasons.) In both cases the $\Omega$-dependence is much weaker than in $d=3$. Whereas there is some weak $\Omega$-dependence for $d=5$, such a dependence is hardly visible for $d=7$. To quantify this diminishing $\Omega$-dependence, in Fig.7b I have plotted the time, $\tau_{\max }$, of the maximum modification as a function of $\Omega$. Whereas one has a $\Omega^{-1}$ dependence in the long-time regime for $d=3$, this is weaker, roughly $\Omega^{-1 / 2}$, for $d=5$ and finally in $d=7$ and $d=9$ there is no visible $\Omega$-dependence. This means that the dynamics in $d=7$ an $d=9$ is dynamic homogeneous in the short-time and the long-time regime. Thus, also the aging dynamics becomes dynamic homogeneous for higher spatial dimension.

The conclusion from these calculations is that the dynamics of the s-FM model becomes dynamic homogeneous in the mean-field limit, $d \gg 1$. However, as pointed out earlier, the mean-field limit holds for all $d>4$ when concerned with static properties. For $d=5$, Fig.7 reveals that the aging dynamics still is heterogeneous, although the shape of an effective distribution of relaxation times is changed relative to $d=3$. 


\section{NHB in the spherical Sherrington-Kirkpatrick model}

As already noted in the preceeding section the dynamic behavior of the s-FM model in $d=3$ and the s-SK model is very similar in the low temperature phase. Concerning the modified response $\chi^{*}(\tau)$ the same holds true, in particular in the limit of large $t_{q}$. The only difference stems from the functions $g(t)$ occuring in the expressions for $\Delta_{0}\left(t_{q}+\tau\right)$ and $R\left(\hat{t}+\tau, \hat{t}+\tau^{\prime}\right)$, eqns.(1619), for the two models. Plotting the two functions $g_{F M}(t)$ and $g_{S K}(t)$ reveals that $g_{F M}(t) \simeq g_{S K}(t / 3)$ in a good approximation for moderate $t$ and asymptotically one has $g_{S K}(t) \simeq\left[(8 \pi)^{3 / 2} /(32 \pi)^{1 / 2}\right] g_{F M}(t) \simeq 12.57 g_{F M}(t)$, cf. eq. (10). For large $\Omega$ small times $\tau$ are relevant and therefore one expects $\Delta \chi_{S K}(\tau) \simeq \Delta \chi_{F M}(\tau)$ to hold with the difference that the maximum modification occurs at $\tau_{\max }^{S K} \simeq 3 \tau_{\max }^{F M}$. On the other hand, for small $\Omega$ long times are most important and all functions $g(t)$ can be replaced by their asymptotic values. Thus, one roughly has $\Delta \chi_{S K}(\tau) \simeq\left[(8 \pi)^{3} /(32 \pi)\right] \Delta \chi_{F M}(\tau)$ and $\tau_{\max }^{S K} \simeq \tau_{\max }^{F M}$. In Fig.8a I have plotted $\Delta \chi(\tau)$ for both models for $\Omega=10^{-4}$ (upper panel) and $\Omega=100$ (lower panel). Apart from a factor of two in the amplitude for the larger frequency the behavior just discussed is recovered.

Fig.8b shows the time of the maximum modification, $\tau_{\max }$ versus frequency $\Omega$. The full line is for the $3 \mathrm{~d}$ s-FM, cf. Fig.4b, and the dashed line is for the s-SK model. Also included is $\left(\tau_{\max }^{S K} / 3\right)$ for $\Omega>1$ (dot-dashed line). From the above discussion it is clear that one just finds the expected behavior: for very small $\Omega$ one has $\tau_{\max }^{S K} \simeq \tau_{\max }^{F M}$ whereas $\tau_{\max }^{S K} \simeq 3 \tau_{\max }^{F M}$ holds for large $\Omega$.

These considerations show that the qualitative behavior of the s-FM model for $d=3$ and the s-SK model are extremely similar in the low temperature phase, including the dynamic heterogeneities. The similarity between the $3 \mathrm{~d}$ s-FM and the s-SK model is well known and it has been argued earlier that the s-SK model does not generically behave like a spin glas, concerning both, the statics and the dynamics [28].

The fact that there are dynamic heterogeneities observable in the s-SK model might appear somewhat astonishing in view of the fact that the s-SK model is a mean-field model. However, this result is not unique. The first observation of dynamic heterogeneous relaxation in a mean-field model was reported in ref. [20]. Also the Monte Carlo simulations on the Sherrington-Kirkpatrick model mentioned above 24] show dynamic heterogeneous behavior. The present results therefore confirm the fact that an identification of dynamic and spatial heterogeneities is not possible in general.

\section{Conclusions}

In the present paper I presented calculations of the response of simple spherical spin models to the field sequence of the nonresonant hole burning experiment, a technique allowing the detection of dynamic heterogeneities if they exist. The calculations were restricted to the generic situation of aging in the low temperature phase. It was assumed that the system is quenched from infinite temperature to $T<T_{c}$ at the beginning, correlations in the initial conditions thus being neglected completely. The equilibrium dynamics will be considered 
in a forthcoming publication.

The main result of the present paper is the fact that the non-equilibrium dynamics of the s-FM model in $d=3$ is dynamic heterogeneous in the aging regime whereas it appears homogeneous in the short-time regime. In higher dimensions, $d \geq 7$, the response becomes dynamic homogeneous also in the aging regime. Therefore, the nature of the aging dynamics changes and in the mean-field limit, $d \gg 1$, the model displays homogeneous dynamics. This is what one would expect as in this limit each spin interacts with an infinite number of neighboring spins. However, for the s-SK model, a simple mean-field spin glass model, a behavior very similar to that of the s-FM model in $d=3$ is found. This explicitly demonstrates that the existence of dynamic heterogenities does not tell us anything about spatial heterogeneities in the system. Furthermore, a strongly heterogeneous dynamics has also been observed in the aging behavior of a short range spin glass model 34] showing strong relations between local responses and correlations. The mere existence of dynamic heterogeneities in a short range spin glass is of course to be expected and here also the spatial aspects of these heterogeneities are of importance.

When considering the spherical two-spin interaction models, it is tempting to associate some disorder with the spherical constraint which forces the equal time correlation function to unity at all times. The lengths of the spins are not static quantities and are random to some extent. This might be viewed as a kind of 'dynamic' disorder and the similarities between the s-FM model in $d=3$ and the s-SK model hint towards the irrelevance of the quenched disorder in the latter when concerned with dynamic quantities. On a speculative level the fact that the dynamics in the s-FM model becomes homogeneous for $d \gg 1$ can be understood from the following argument. Assuming the existence of an effective distribution of relaxation rates at a given instant of time, i.e. for a given distribution of $s_{i}(t)^{2}$ the width of this distribution is expected to decrease with an increasing number of nearest neighbor interactions and consequently the dynamics becomes more homogeneous. This of course does not mean that the response decays in an exponential way for large $d$ because the considered distribution is not a static quantity. Notice that this argument also implies that the life-time of the heterogeneities is finite. Unfortunately, NHB does not allow to determine this life-time. It is, however, important to point out that the argument concerning the increasing number of neighbors, though appealing with respect to the s-FM model, has important drawbacks. Taking it serious in case of the s-SK model would predict homogeneous dynamics in contrast to what is observed.

Although the s-FM model is a typical model for phase-ordering kinetics, there is no obvious relationship between the observed dynamic heterogeneities and the domain size distribution in a coarsening system. A possible way to investigate such a relationship could be to perform calculations on the Ising model using spatially varying magnetic fields. It would also be interesting to perform an analysis along the lines of refs. 34, 35] in order to see whether the dynamic heterogeneities in a coarsening system behave similar to those observed in glassy systems. Furthermore, such calculations would allow to investigate the dependence on spatial dimension and therefore to check whether the one observed in the present paper for the s-FM model also is found for other domain-coarsening models. 
In summary, I have shown that heterogeneous aging can be observed in the lowtemperature phase of the spherical model of a ferromagnet. The aging dynamics becomes homogeneous on increase of the spatial dimension despite the fact that no qualitative change in the two-time quantities like the correlation function or the response is observed. Quenched disorder does not play any significant role with respect to heterogeneities in spherical models.

\section{Acknowledgements}

I thank R. Böhmer, G. Hinze, U. Häberle and R. Schilling for fruitful discussions. This work was supported by the DFG under contract Di693/1-1. 


\section{References}

[1] for reviews see: K. Binder and A.P. Young; Rev. Mod. Phys. 58801 (1986)

C.A. Angell; J. Phys. Chem. Solids 8863 (1988)

U. Mohanty; Adv. Chem. Phys. 8989 (1995)

M.D. Ediger, C.A. Angell and S.R. Nagel; J. Phys. Chem. 10013200 (1996)

[2] M.D. Ediger; Annu. Rev. Phys. Chem. 5199 (2000)

H.Sillescu; J. Non-Cryst. Solids 24381 (1999)

R. Böhmer; Current Opinion in Solid State and Material Science 3378 (1998)

R. Böhmer, R.V. Chamberlin, G. Diezemann, B. Geil, A. Heuer, G. Hinze, S.C.

Kuebler, R. Richert, B. Schiener, H. Sillescu, H.W. Spiess, U. Tracht and M. Wilhelm;

J. Non-Cryst. Solids 235-237 1 (1998)

[3] K. Schmidt-Rohr and H.W. Spiess; Phys. Rev. Lett. 663020 (1991)

[4] M.T. Cicerone and M.D. Ediger; J. Chem. Phys. 1035684 (1995)

[5] E. Vidal- Russell and N.E. Israeloff; Nature 408695 (1999)

[6] L.A. Deschenes and D. Vanden Bout; Science 292255 (2001)

[7] H. Sillescu, R. Böhmer, G. Hinze and G. Diezemann; J. Non-Cryst. Solids 307-310 $16(2002)$

[8] A. Heuer, M. Wilhelm, H. Zimmermann and H.W. Spiess; Phys. Rev. Lett. 752851 (1995)

[9] R. Böhmer, G. Hinze, G. Diezemann, B. Geil and H. Sillescu; Europhys. Lett. 3655 (1996)

[10] A. Heuer; Phys. Rev. E 56730 (1997)

[11] G. Diezemann; J. Chem. Phys. 10710112 (1997)

[12] U. Tracht, M. Wilhelm, A. Heuer, H. Feng, K. Schmidt-Rohr and H.W. Spiess; Phys. Rev. Lett. 812727 (1998)

[13] S.A. Reinsberg, X.H. Qiu, M. Wilhelm, H.W. Spiess and M.D. Ediger; J. Chem. Phys. 1147299 (2001)

[14] S.A. Reinsberg, A. Heuer, B. Doliwa, H. Zimmermann and H.W. Spiess; J. NonCryst. Solids 307-310 208 (2002)

[15] B. Schiener, R.Böhmer, A. Loidl and R.V. Chamberlin; Science 274752 (1996)

[16] B. Schiener, R.V. Chamberlin, G. Diezemann and R. Böhmer; J. Chem. Phys. 107 7746 (1997) 
[17] O. Kircher, B. Schiener and R. Böhmer; Phys. Rev. Lett. 814520 (1998)

[18] R. Richert and R. Böhmer; Phys. Rev. Lett. 834337 (1999)

[19] R.V. Chamberlin; Phys. Rev. Lett. 835134 (1999)

[20] L.F. Cugliandolo and J.L. Iguain; Phys. Rev. Lett. 853448 (2000)

[21] R. Kubo, M. Toda and N. Hashitsume Statistical Physics II Springer, BerlinHeidelberg-New York (1992)

[22] G. Diezemann; Europhys. Lett. 53604 (2001)

[23] G. Diezemann and R. Böhmer; Phys. Rev. Lett. 87129602 (2001)

[24] L.F. Cugliandolo and J.L. Iguain; Phys. Rev. Lett. 87129603 (2001)

[25] L.F. Cugliandolo and D.S. Dean; J. Phys. A 284213 (1995)

L.F. Cugliandolo and D.S. Dean; J. Phys. A 28 L453 (1995)

[26] A.J. Bray; Adv. Phys. 43357 (1994)

[27] C. Godreche and J.M. Luck; J. Phys. A 339141 (2000)

[28] W. Zippold, R. Kühn and H. Horner; Eur. Phys. J. B 13531 (2000)

[29] J.P. Bouchaud, L.F. Cugliandolo, J. Kurchan and M. Mezard; in Spin glasses and random fields, Ed. A.P. Young, World Scientific, Singapore (1998)

[30] A. Barrat; Phys. Rev. E 573629 (1998)

L. Berthier, J.L. Barrat and J. Kurchan; Eur. Phys. J. B 11635 (1999)

[31] L. Berthier, L.F. Cugliandolo and J.L. Iguain; Phys. Rev. E 63051302 (2001)

[32] T.H. Berlin and M. Kac; Phys. Rev. 86821 (1952)

[33] F. Corberi, E. Lippiello and M. Zannetti; Phys. Rev. E 65046136 (2002)

[34] H.E. Castillo, C. Chamon, L.F. Cugliandolo and M.P. Kennett; Phys. Rev. Lett. 88 $237201(2002)$

[35] A. Montanari and F. Ricci-Tersenghi; Phys. Rev. Lett. 90017203 (2003) 


\section{Figure captions}

Fig.1 : The response function $R\left(\tau+t_{w}, t_{w}\right)$ for the s-FM model in the ferromagnetic phase, $T<T_{c}$, for $t_{w}=10^{2}, 10^{3}, 10^{4}, 10^{5}$ (from upper to lowest line). Upper panel: $d=3$, middle panel: $d=5$, lower panel: $d=7$.

Fig.2 : The field sequence for the nonresonant hole burning (NHB) experiment: A time $t_{q}$ after a quench from $T=\infty$ one or more cycles of a strong sinusoidal field $h_{p}(t)=h_{p} \sin \left(\Omega\left(t-t_{q}\right)\right)$ are applied. After a waiting time $t_{w}$ the response to an infinitesimally small field is monitored.

Fig.3 : a: The modification of the response, $\Delta \chi(\tau)$ vs. $\tau$ for the s-FM, $d=3$. Upper panel: $\Omega=0.1$; lower panel: $\Omega=10$. The time after the quench from infinite temperature, $t_{q}$, is chosen as $t_{q}=0,10,10^{6}$.

b: $\Delta \chi_{\max , s c .}=\Delta \chi_{\max }\left(t_{q}\right) / \Delta \chi_{\max }\left(t_{q}=10^{-6}\right)$ versus $t_{q}$ for $\Omega=0.01,0.1,10$.

c: $\Delta \chi(\tau)$ versus $\tau$ for $\Omega=0.1$ and $t_{q}=10^{6}$ for $N=1$ (full line), $N=5$ (dashed line) and $N=10$ (dotted line). The curves for $N=5$ and $N=10$ are hardly to distinguish.

d: $\Delta \chi_{\max , s c .}=\Delta \chi_{\max }(N) / \Delta \chi_{\max }(N=1)$ as a function of the number of cycles of the sinusoidal field, $N\left(t_{q}=10^{6}\right)$.

Fig.4 : a: $\Delta \chi(\tau)$ vs. $\tau$ for $t_{q}=10^{6}, t_{w}=0, d=3$ and $\log (\Omega)=1,0,-1,-2,-3,-4$ (from left to right).

b: Full line: $\tau_{\max }$ vs. $\Omega$, dashed line: $\Omega \tau_{\max }$ vs. $\Omega$ for $t_{q}=10^{6}, t_{w}=0$. Additionally shown as the dot-dashed line is $\tau_{\max }$ vs. $\Omega$ for $t_{q}=1$ and $t_{w}=0$, demonstrating that the behavior does not depende on $t_{q}$.

Fig.5 : a: $\Delta \chi\left(\tau_{\max }\right)_{n o r m}$ vs. the scaled waiting time $\Omega t_{w}$ for $\Omega=10^{-4}, 1,5,10,25,50,10^{2}$ (from left to right).

b: Characteristic decay time $\tau_{K}$ of a Kohlrausch-fit of the from $\Delta \chi\left(\tau_{\text {max }}\right)_{\text {norm. }}=$ $\exp \left\{-\left(t_{w} / \tau_{K}\right)^{\beta_{K}}\right\}\left(t_{q}=10^{6}\right)$. The stretching parameter is approximately constant, $\beta_{K} \simeq 0.9$. The dot-dashed line represents $\tau_{\max }$ for $t_{w}=0$ for comparison, cf. Fig. $4 \mathrm{~b}$.

Fig.6 : a: $\Delta \chi(\tau)$ vs. temperature for $T=0,0.1,0.2,0.3,0.4,0.5$ for the burn frequencies given in the respective panels. The remaining parameters are $t_{q}=10^{6}, t_{w}=0$ and $d=3$.

b: The value of the maximum modification, $\Delta \chi\left(\tau_{\max }\right)$, vs. $1-T / T_{c}$. The other parameters are the same as in a.

Fig.7 : a: $\Delta \chi(\tau)$ vs. $\tau$ for burn frequencies $\Omega=1,10,20,50,100$ for $d=5$ (upper panel) and $d=7$ (lower panel). The remaining parameters are $t_{q}=10^{6}$ and $t_{w}=0$.

b: The time of the maximum modification, $\tau_{\max }$, for $d=3, d=5$ and $d=7$ vs. $\Omega$. In the upper panel $\tau_{\max }$ is shown and in the lower one the product $\Omega \tau_{\max }$. Full lines are for $t_{q}=10^{6}$ and the dot-dashed lines for $t_{q}=0$. 
Fig.8 : a: $\Delta \chi(\tau)$ vs. $\tau$ for the 3-dimensional s-FM (full line) and the s-SK model (dashed line). Upper panel: $\Omega=10^{-4}$ and the factors muliplying $\Delta \chi(\tau)$ are $N(S K)=32 \pi$ and $N(F M)=(8 \pi)^{3}$. Lower panel: $\Omega=10^{2}$. The remaining parameters are $t_{q}=10^{6}$ and $t_{w}=0$.

b: The time of the maximum modification, $\tau_{\max }$, for the $3 \mathrm{~d}$ s-FM model (c.f. Fig.4b, full line) and the s-SK model (dashed line) vs. $\Omega$ for the same parameters as in a. Also included is $\tau_{\max }^{S K} / 3$ (dot-dashed line) for $\Omega>1$. 


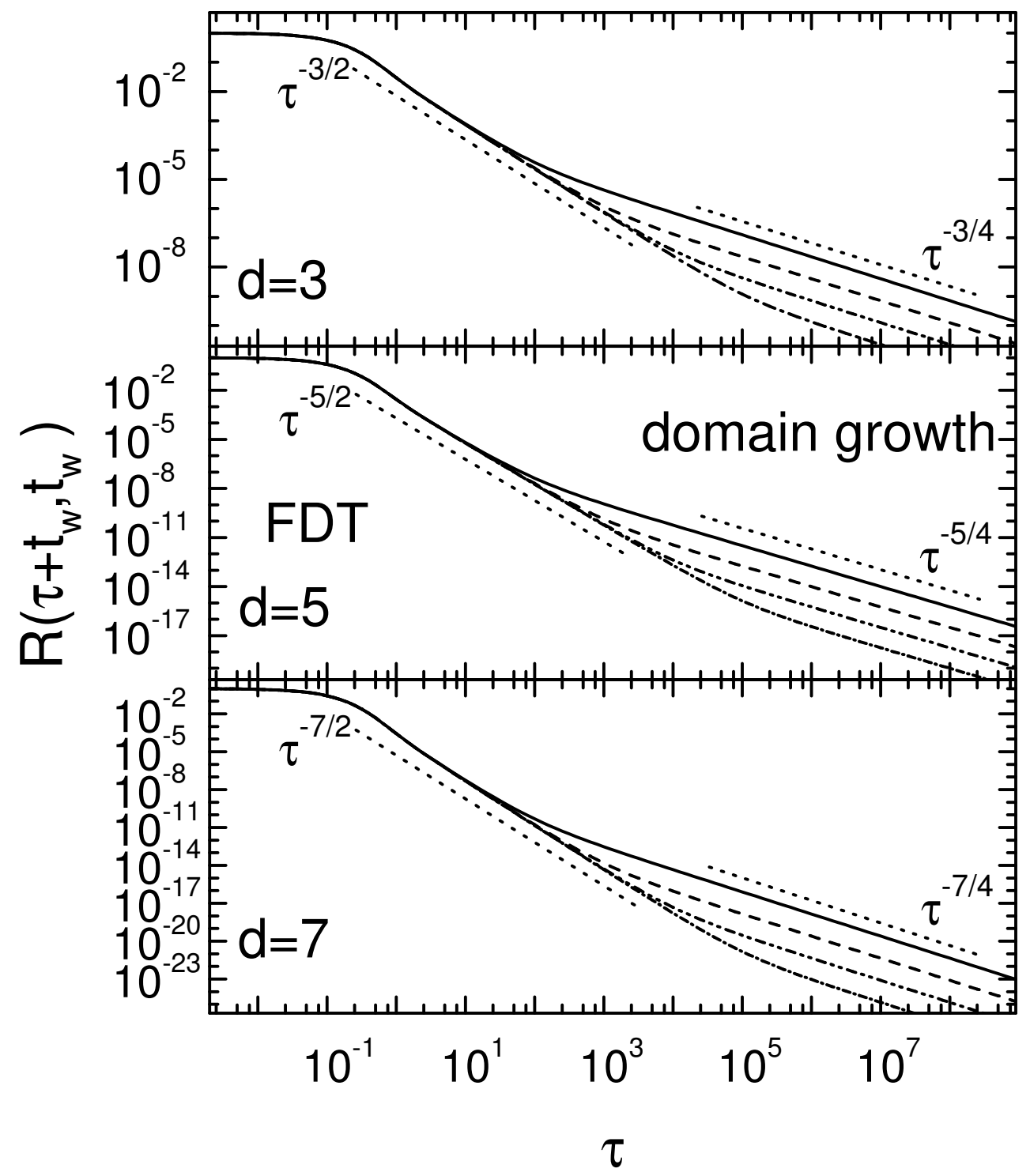

G. Diezemann; Fig.1 


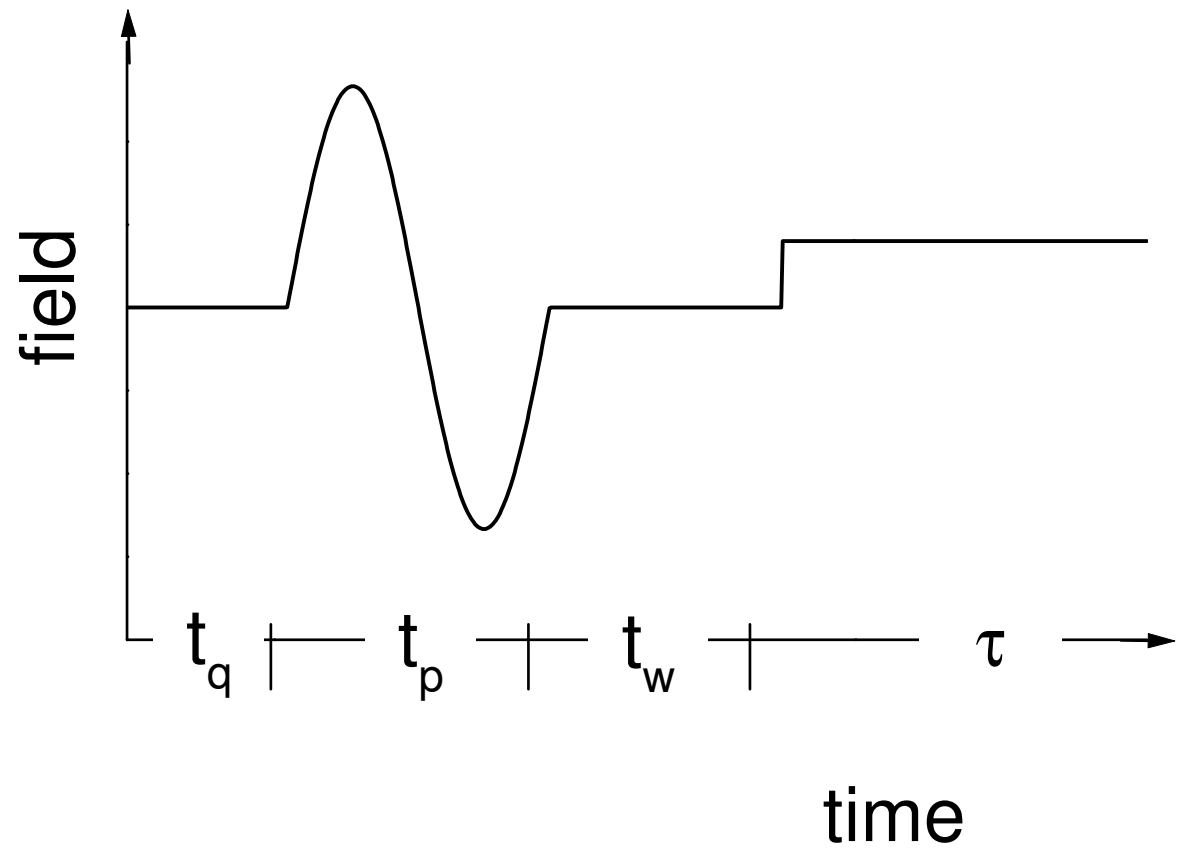

G. Diezemann; Fig.2 


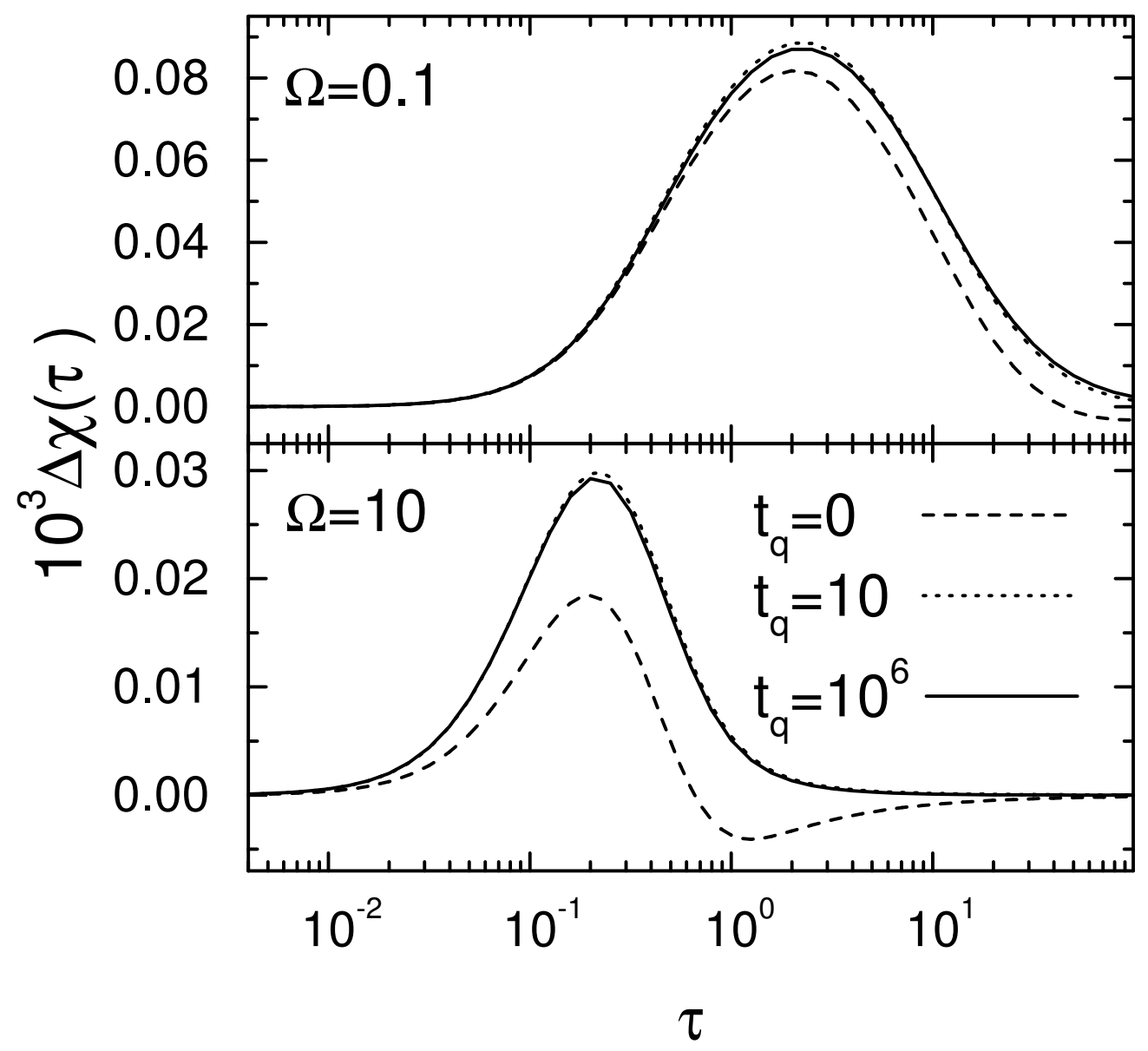

G. Diezemann; Fig.3a 


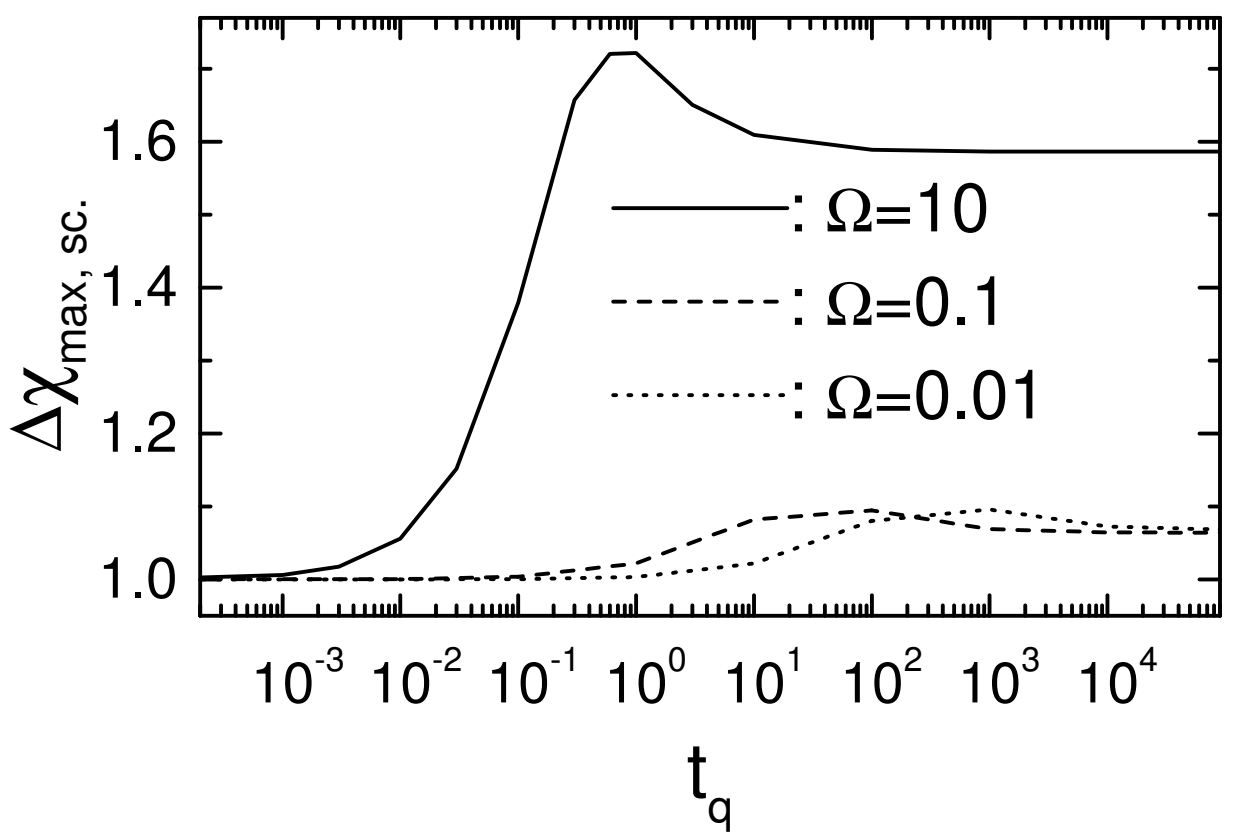

G. Diezemann; Fig.3b 


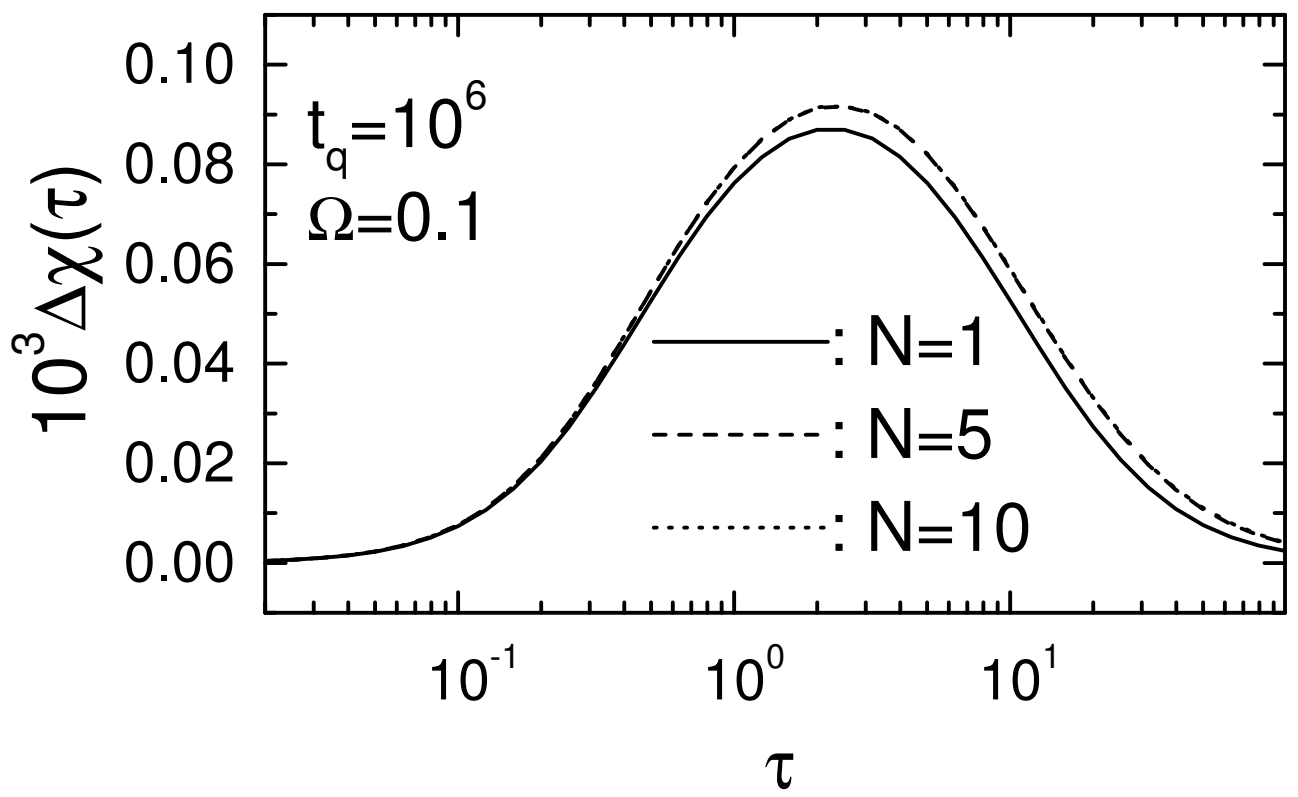

G. Diezemann; Fig.3c 


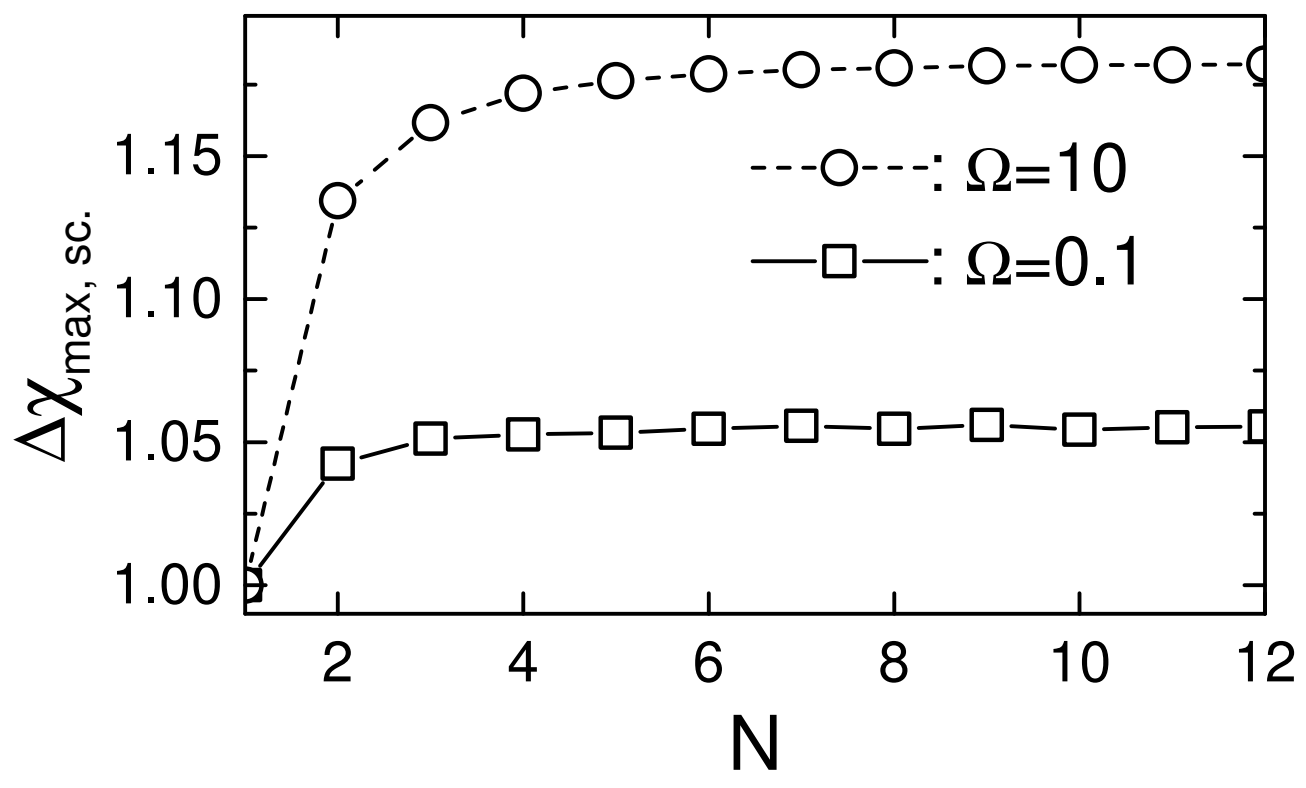

G. Diezemann; Fig.3d 


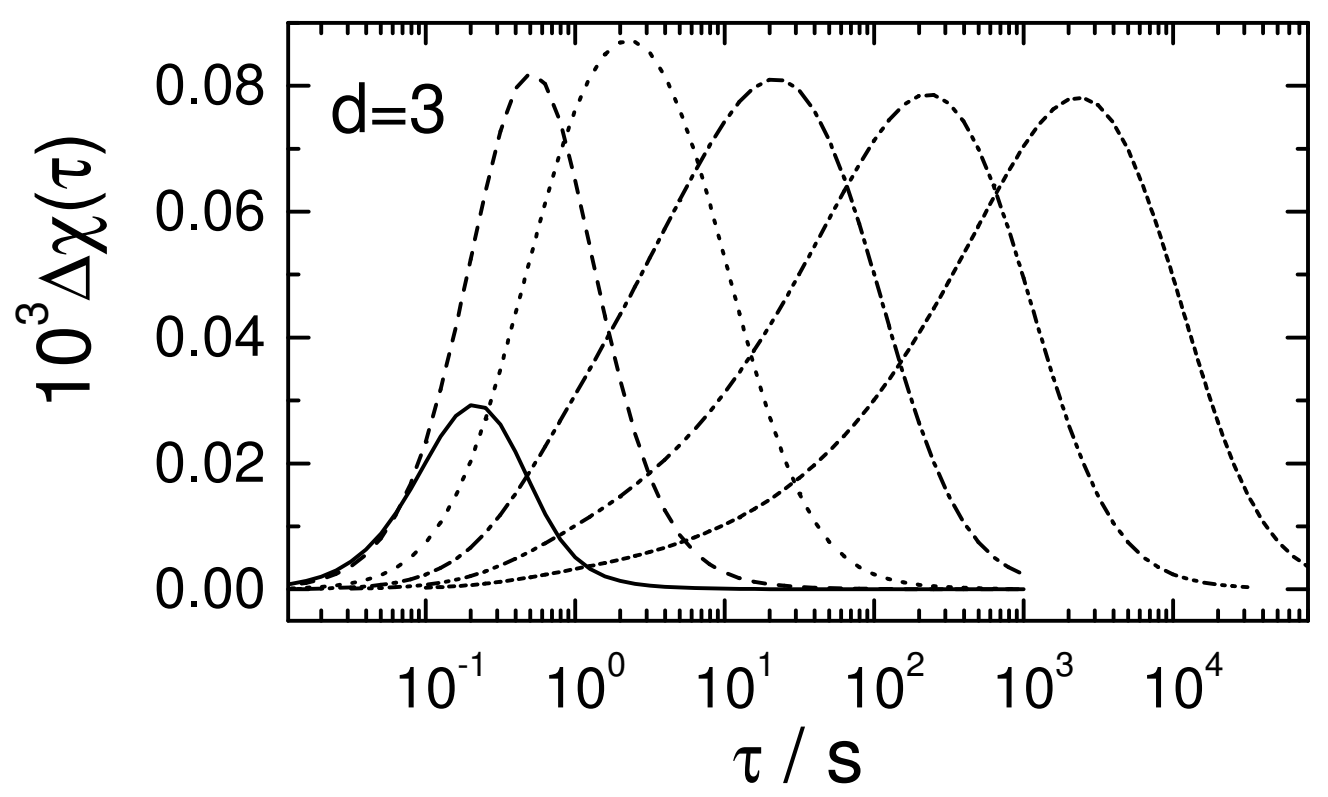

G. Diezemann; Fig.4a 


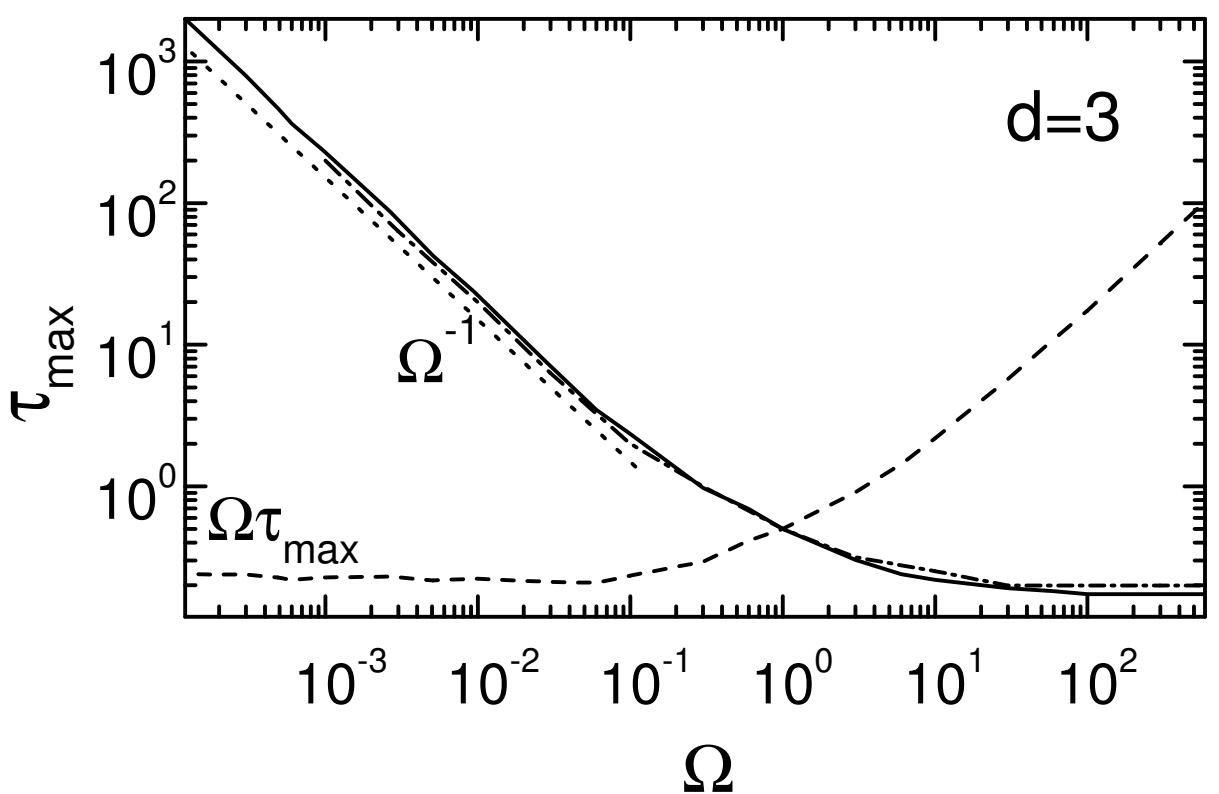

G. Diezemann; Fig.4b 


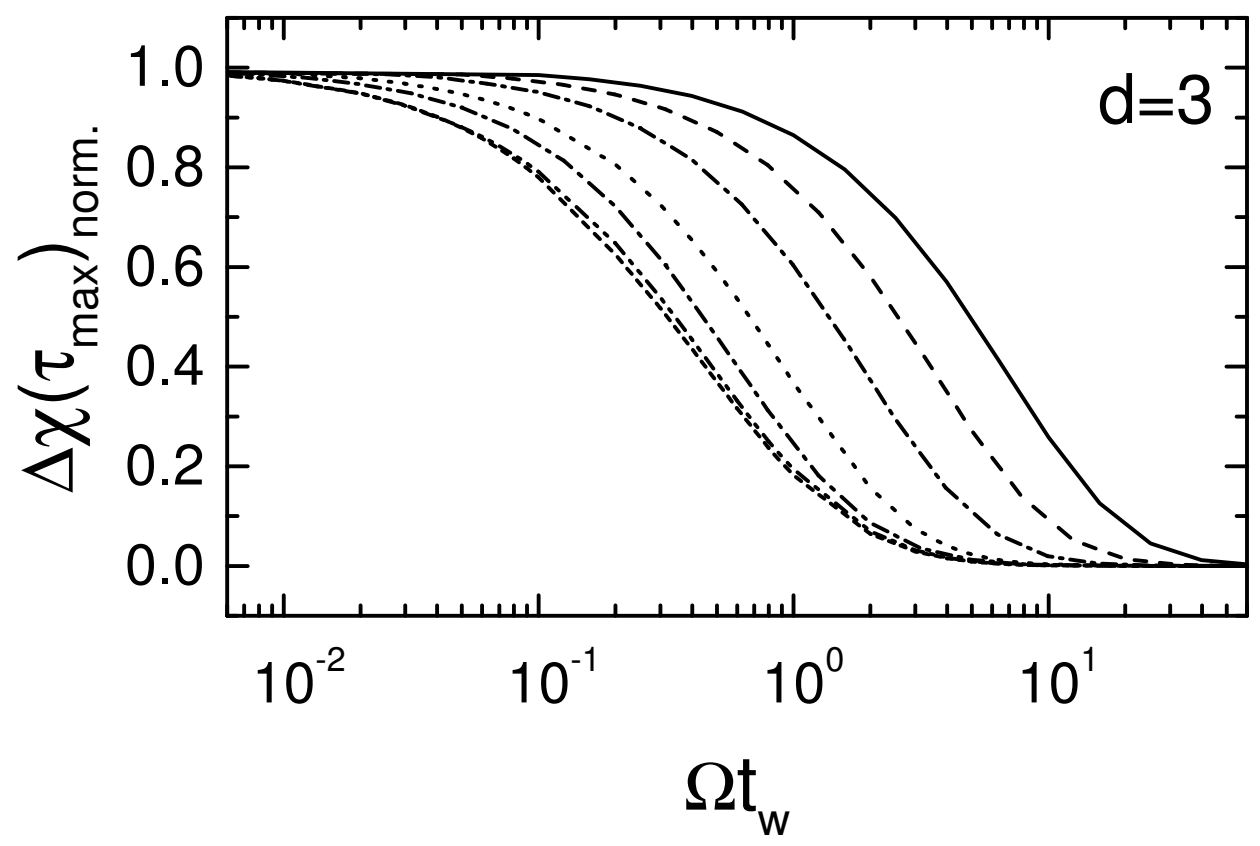

G.Diezemann; Fig.5a 


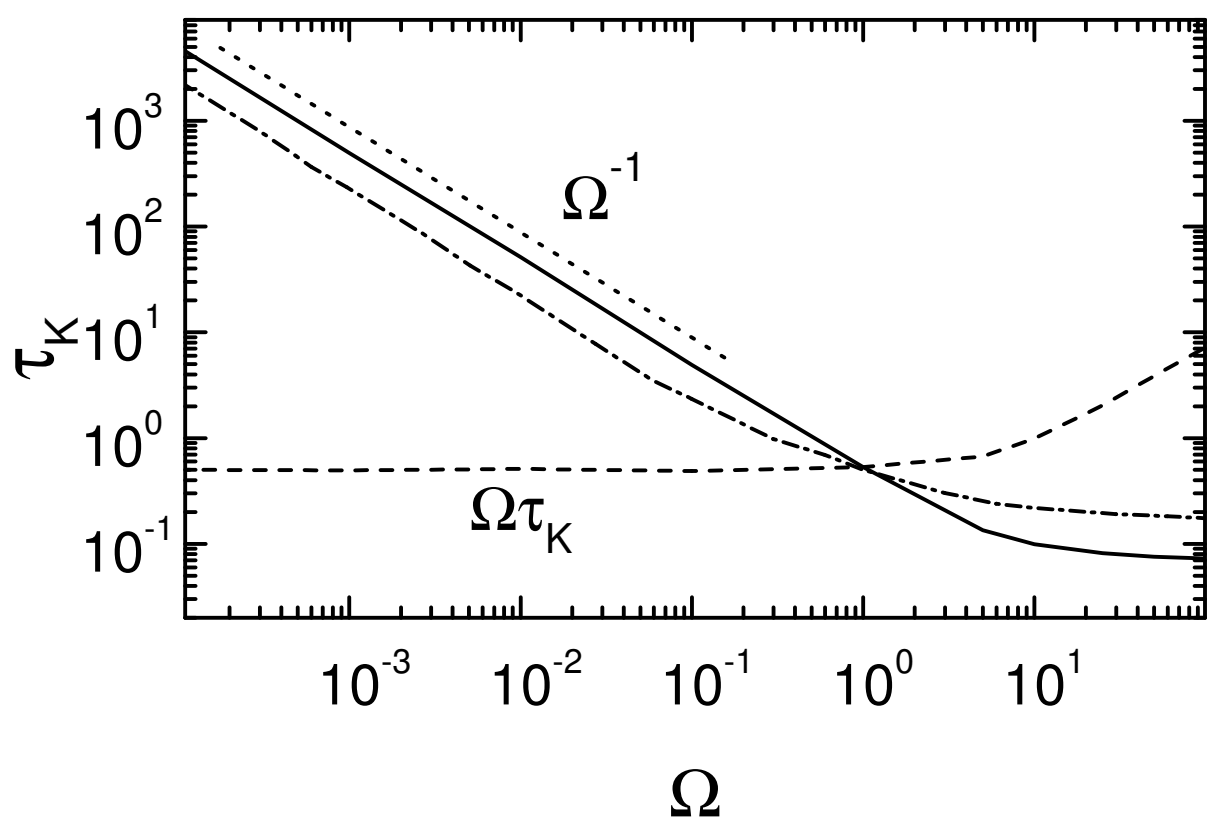

G.Diezemann; Fig.5b 


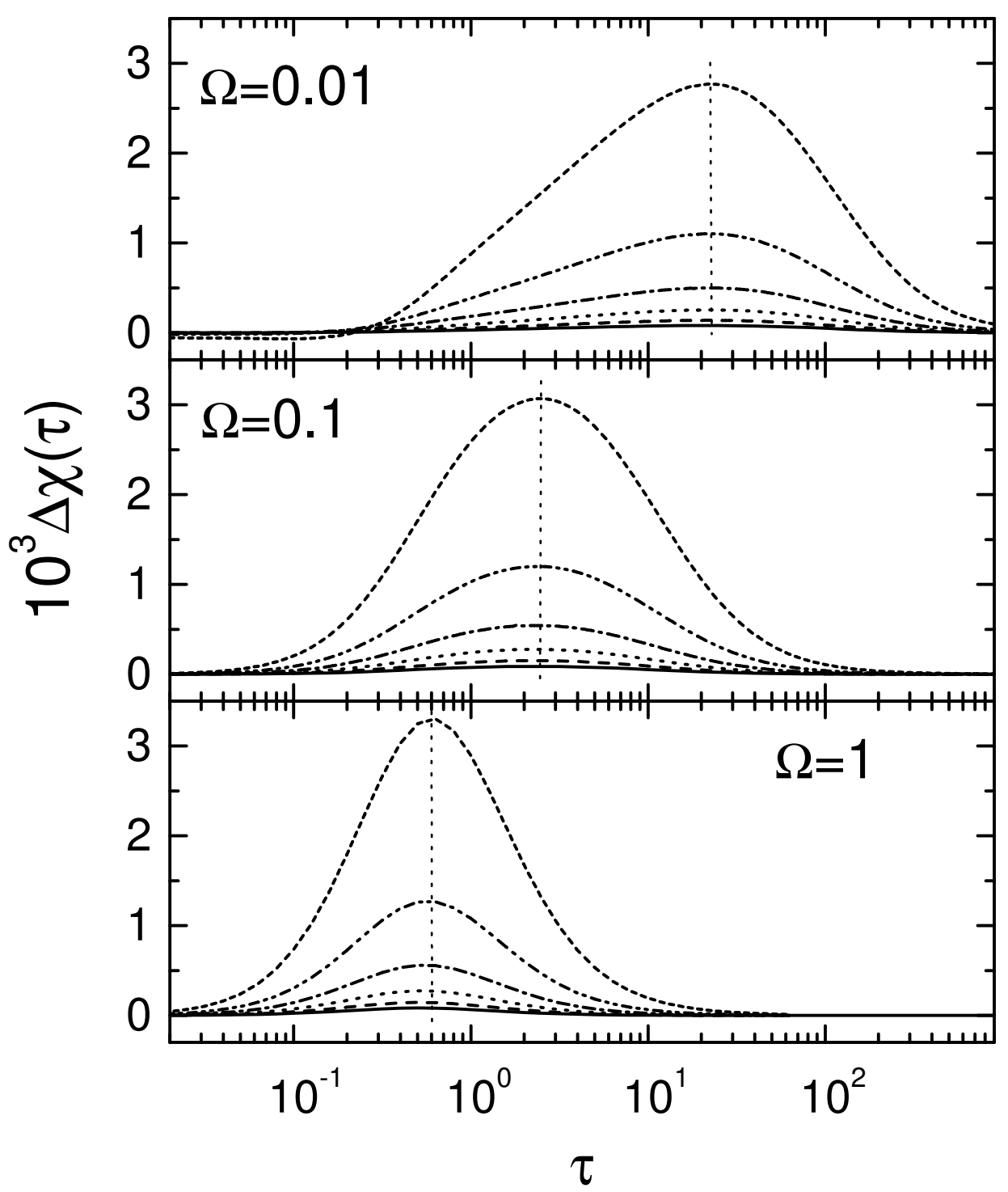

G. Diezemann; Fig.6a 


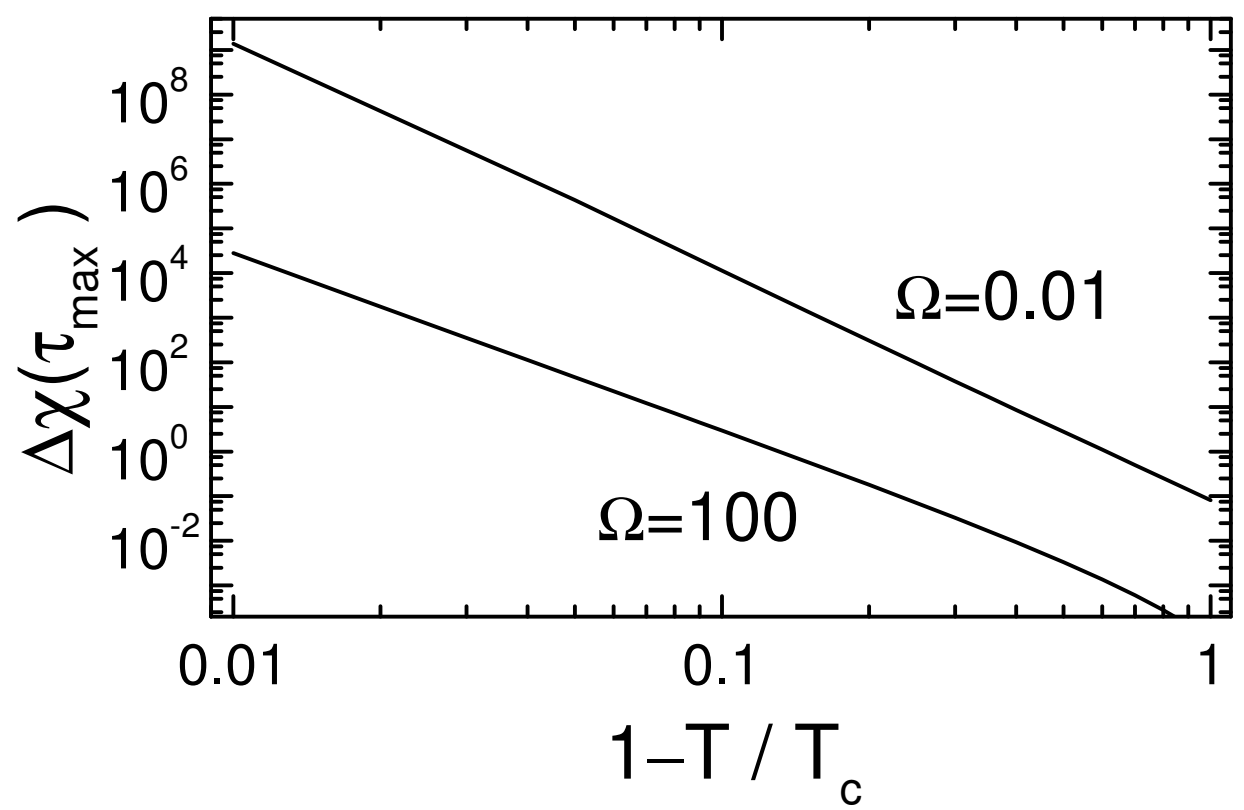

G. Diezemann; Fig.6b 


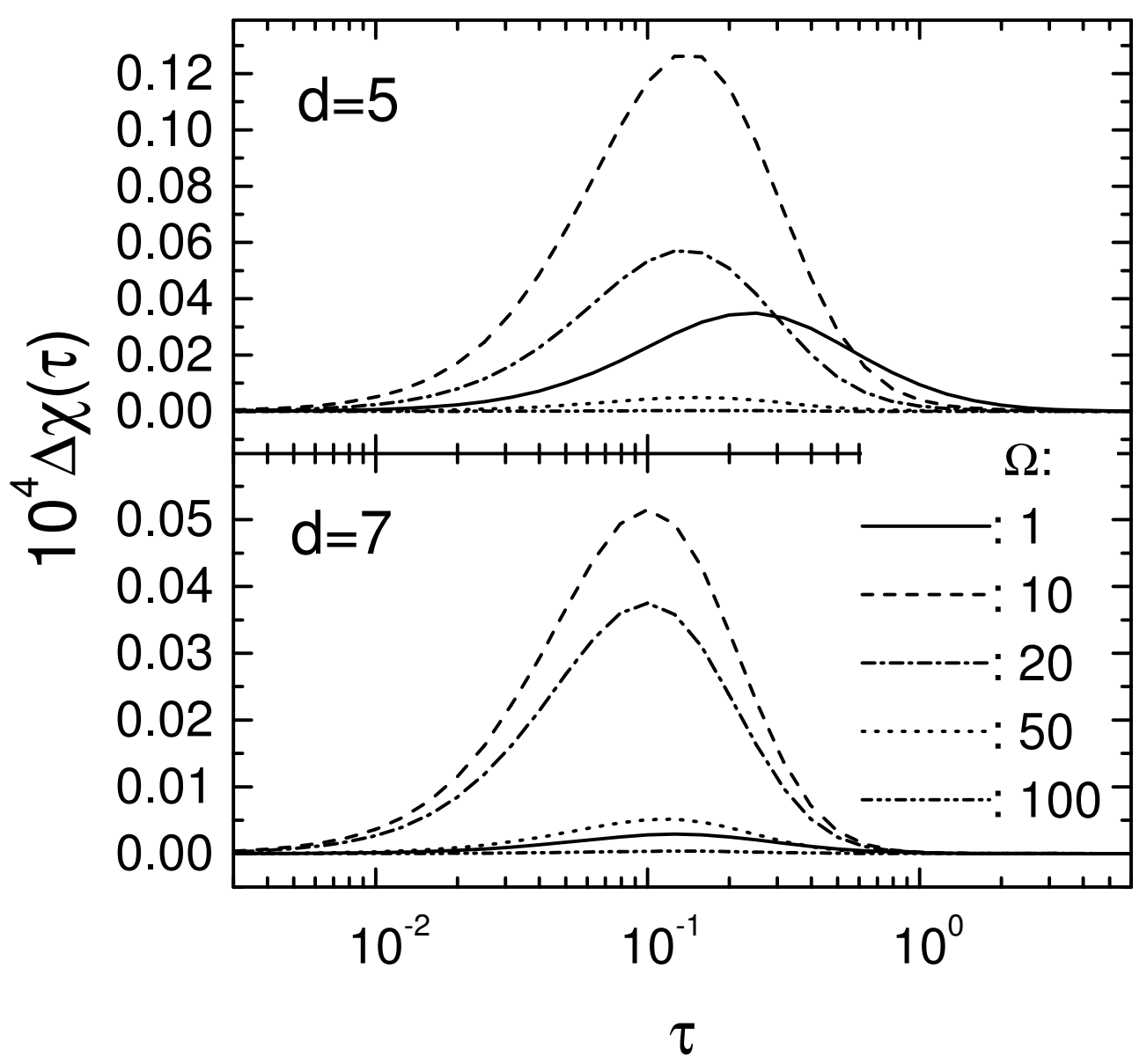

G. Diezemann; Fig. 7a 


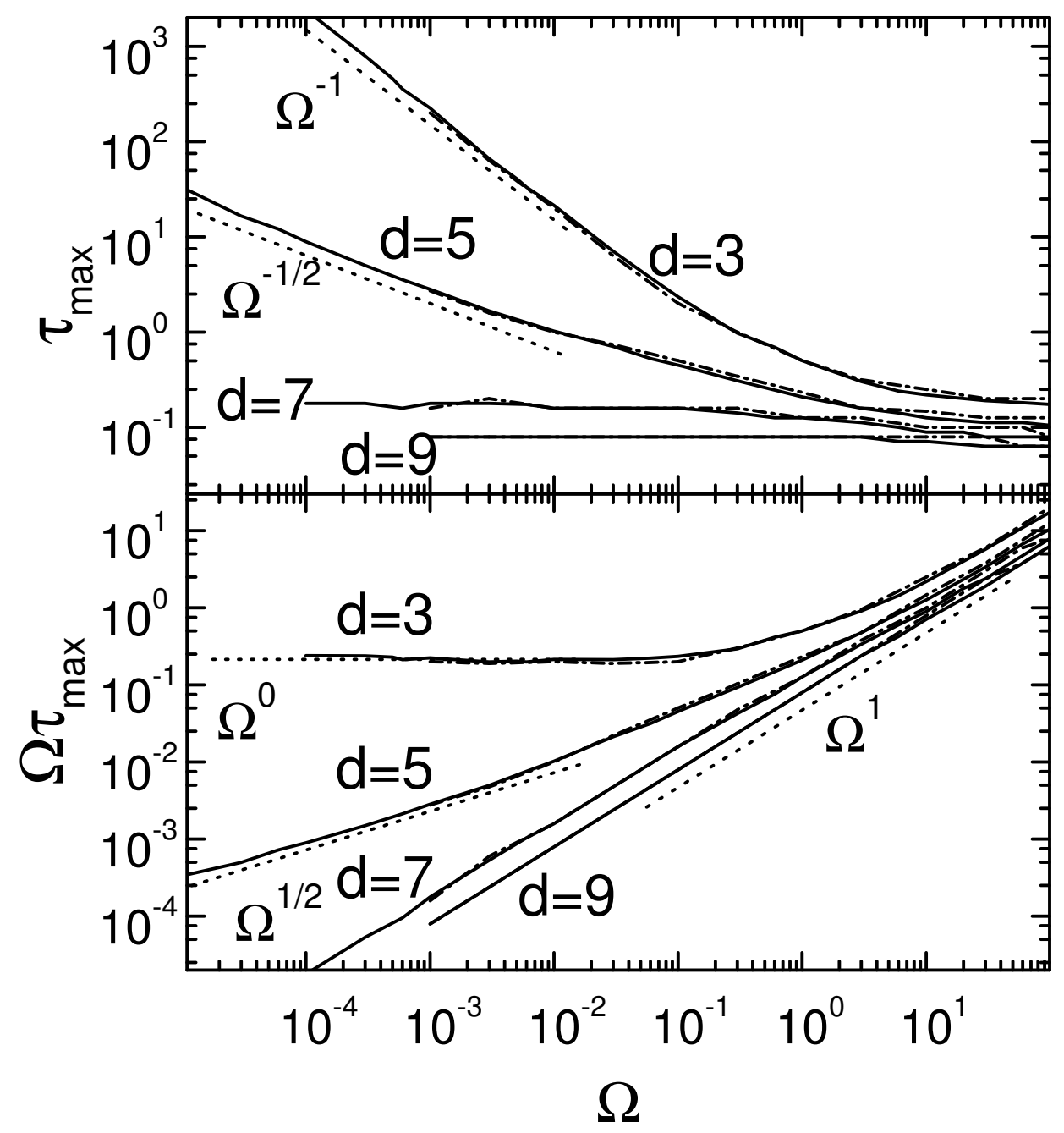

G. Diezemann; Fig. 7b 

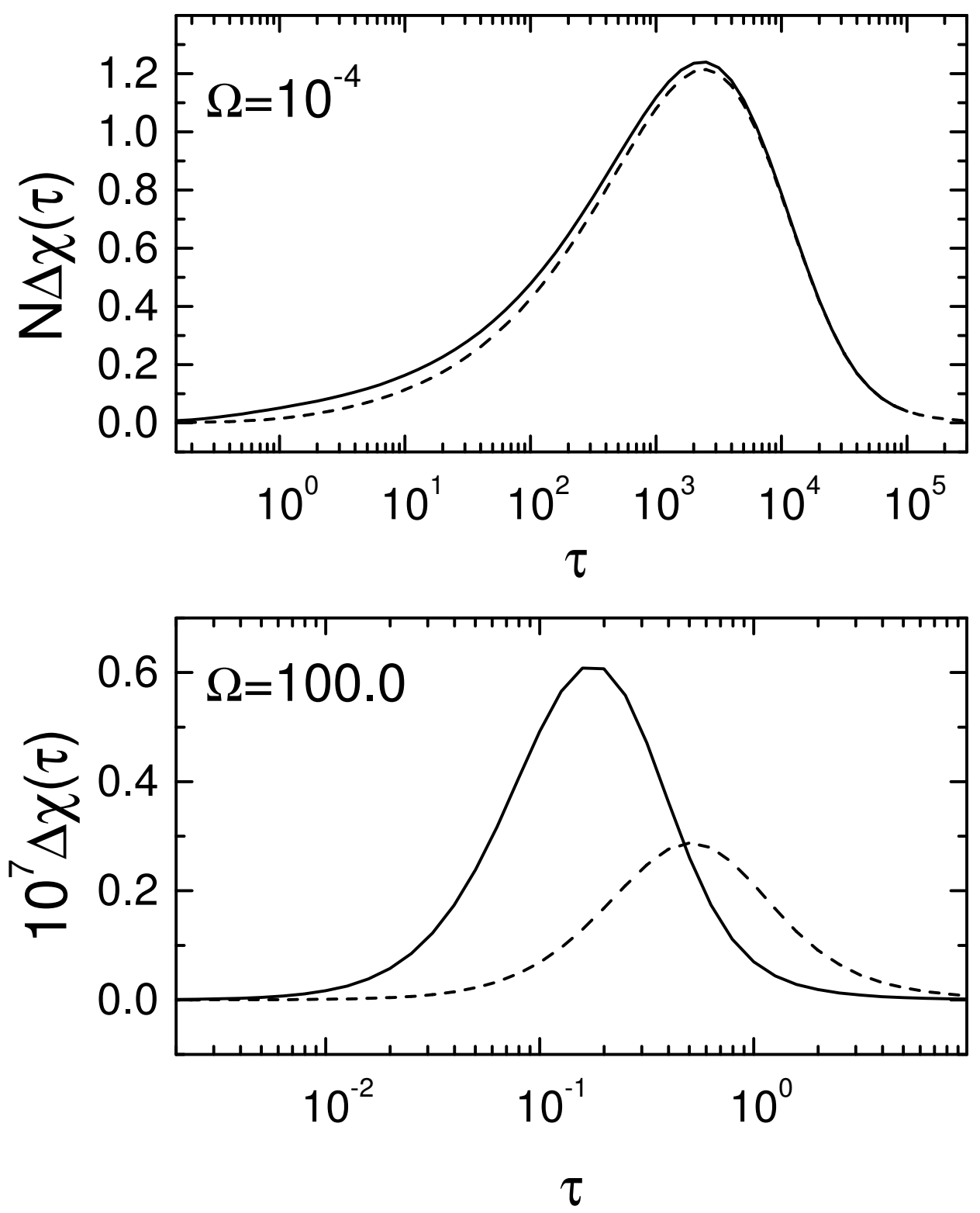

G. Diezemann; Fig. 8a 


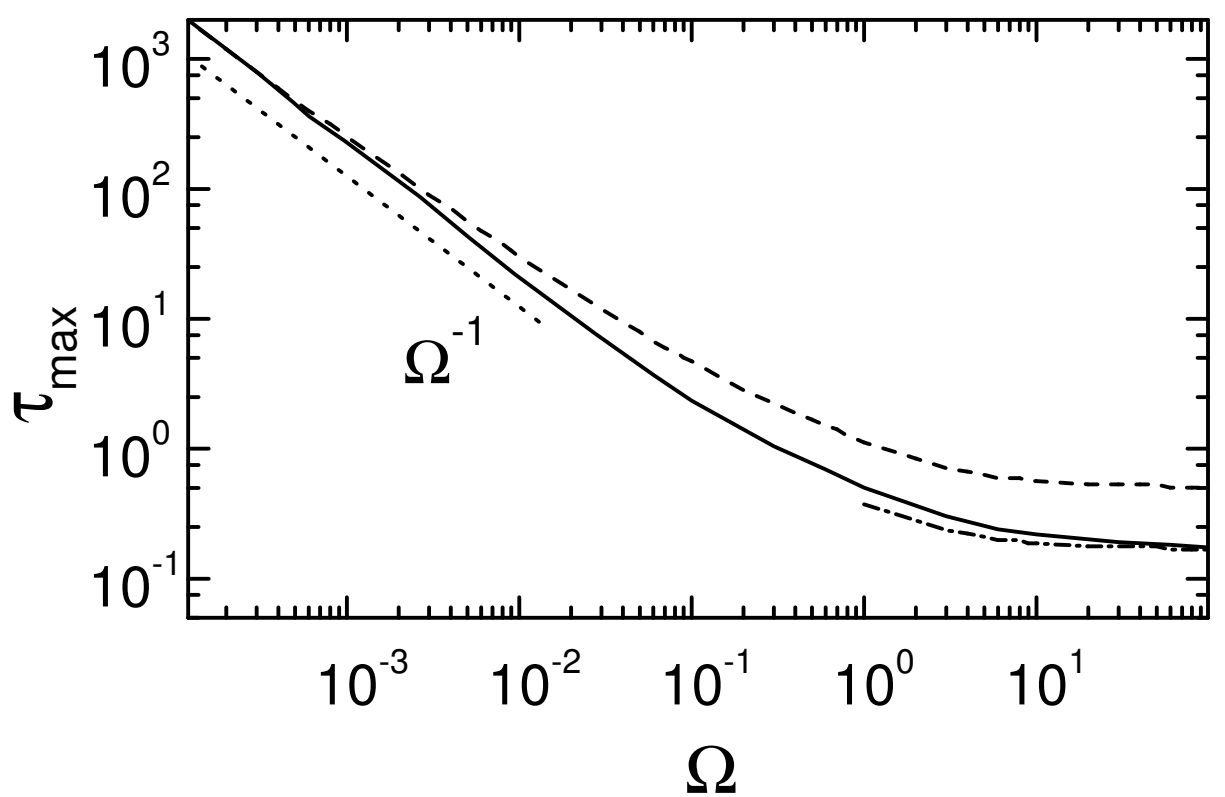

G. Diezemann; Fig. 8b 